\title{
Un modelo social cognitivo de intenciones emprendedoras en estudiantes universitarios
}

\author{
Ana Lanero ${ }^{1 *}$, José-Luis Vázquez ${ }^{1}$ y Alfredo Muñoz-Adánez ${ }^{2}$ \\ ${ }^{1}$ Departamento de Dirección y Economía de la Empresa, Universidad de León \\ 2 Sección Departamental de Psicología Social, Universidad Complutense de Madrid
}

\begin{abstract}
Resumen: Este trabajo analiza las limitaciones de la literatura previa sobre intenciones emprendedoras de estudiantes universitarios y propone un modelo de elección de carreras emprendedoras basado en la Teoría Socia Cognitiva de Carrera (TSCC) de Lent, Brown y Hackett (1994, 2000). Particularmente, el estudio hipotetiza una relación secuencial directa entre intereses vocacionales, intenciones y conductas emprendedoras, desencadenada a su vez por las creencias de autoeficacia y expectativas de resultados extrínsecos e intrínsecos atribuidos a la creación de empresas. En la puesta a prueba del modelo, se describe un estudio realizado con 400 estudiantes universitarios de distintas áreas de conocimiento. Los resultados del trabajo, obtenidos mediante la técnica PLS (Partial Least Squares), confirman una relación positiva entre intereses, intenciones y conductas emprendedoras tempranas y erigen la autoeficacia emprendedora como el principal desencadenante de dicha secuencia de metas. Por su parte, las expectativas de resultados intrínsecos se asocian con los intereses vocacionales por la creación de empresas mientras que las recompensas esperadas a nivel extrínseco no influyen en el modelo. Los resultados tienen importantes implicaciones para el diseño de programas de apoyo a la iniciativa emprendedora en entornos universitarios.

Palabras clave: Iniciativa emprendedora; intención emprendedora; Teoría Social Cognitiva de Carrera (TSCC); elección de carrera; autoeficacia emprendedora; estudiantes universitarios; ecuaciones estructurales.
\end{abstract}

\section{Introducción}

A lo largo de las últimas décadas, el desempleo, la inestabilidad laboral y la sobrecualificación han pasado a representar los descriptores más representativos del proceso de inserción laboral de los jóvenes recién titulados universitarios en España (Blanch Ribas, 2005; García-Montalvo y Peiró, 2009, 2011; Peiró, Sora y Caballer, 2012) y otros países de Europa (Eurostat, 2011; Organización para la Cooperación y el Desarrollo Económicos, 2011). Como respuesta a dicha circunstancia, los gobiernos contemporáneos han comenzado a reivindicar el potencial de la iniciativa emprendedora como fuente de empleo, riqueza y bienestar social en las economías modernas, encomendándose a los sistemas de educación superior la responsabilidad de canalizar las nuevas generaciones de población activa hacia propósitos emprendedores acordes con las nuevas necesidades de los sectores productivos (Comisión Europea, 2006; Dirección General de Empresa e Industria, 2008, 2012a). Todo ello se ha traducido en algunas iniciativas pioneras en las universidades de nuestro entorno, encaminadas fundamentalmente a detectar las inquietudes emprendedoras surgidas entre los estudiantes y tratar de traducirlas en nuevas empresas para el mercado

* Dirección para correspondencia [Correspondence address]: Ana Lanero Carrizo. Facultad de Ciencias Económicas y Empresariales. Campus de Vegazana s/n; 24071 León (España).

E-mail: ana.lanero@unileon.es
Title: A social cognitive model of entrepreneurial intentions in university students.

Abstract: This work analyzes the limitations of previous literature on entrepreneurial intentions of university students and proposes a model of entrepreneurial career choice based on Social Cognitive Career Theory (SCCT) by Lent, Brown and Hackett $(1994,2000)$. Particularly, the study hypothesizes a sequential direct path between entrepreneurial vocational interests, intentions and nascent behaviors, which in turn would be triggered by the beliefs of self-efficacy and outcome expectations attributed to business start-up at both extrinsic and intrinsic levels. To test the hypotheses of the model, an empirical study carried out with 400 university students within different knowledge areas is described. The results obtained, using the PLS (Partial Least Squares) technique, confirm a positive direct link between entrepreneurial interests, intentions and nascent behaviors, while perceived entrepreneurial self-efficacy predicts such goal sequence. Otherwise, intrinsic outcome expectations are related to vocational interest in business startup, whereas expected extrinsic rewards do not play any important role in the model. These findings have important implications for the design of entrepreneurship support programs in university environments.

Key words: Entrepreneurship; entrepreneurial intentions; Social Cognitive Career Theory (SCCT); career choice; entrepreneurial self-efficacy; university students; structural equations.

(Corduras, Urbano, Rojas y Martínez, 2008; Placer y Vázquez, 2005; Ruíz, Solé y Veciana, 2004).

A pesar de los esfuerzos en esta línea, lo cierto es que la implicación de los graduados europeos en iniciativas empresariales está muy lejos de la deseada, especialmente cuando se les compara con otros países, como Estados Unidos (Dirección General de Empresa e Industria, 2012b; Ronald, Kelley, Kew, Herrington y Vorderwülbecke, 2013). Particularmente, en España se estima que menos de un 4\% de recién titulados universitarios optan por la creación de empresas en su primer empleo (Alemany, Álvarez, Planellas y Urbano, 2011; García-Montalvo y Peiró, 2011; Hernández y Corduras, 2013), mientras que el trabajo en organizaciones ya establecidas o en la Administración Pública representa la opción predilecta (Guerrero, Rialp y Urbano, 2008; Toledano y Urbano, 2008; Veciana, Aponte y Urbano, 2005).

En tales circunstancias, surge la necesidad de avanzar hacia un mejor conocimiento de los procesos individuales y contextuales implicados en el desarrollo de la iniciativa emprendedora en la etapa universitaria, de cara a lograr una mayor eficiencia en la disposición de recursos de apoyo a tal fin a través de la educación superior. En línea con dicho propósito, la mayor parte de aproximaciones previas al estudio de la iniciativa emprendedora en colectivos universitarios han tomado como fundamentación teórica modelos generales extraídos de la literatura sobre creación de empresas. A este respecto, el enfoque empleado por excelencia hoy en día dentro de una perspectiva cognitiva concibe el establecimiento de un negocio como un acto propositivo que deriva 
de la formación de intenciones al respecto. El fundamento de este postulado proviene de la literatura psicológica en la que la intencionalidad ocupa un puesto destacado en la motivación de la conducta planificada orientada a metas, especialmente cuando ésta se desarrolla a largo plazo (Ajzen, 1987, 1991, 2001; Ajzen y Fishbein, 1980).

Los modelos teóricos más frecuentemente empleados dentro de esta corriente remiten al Modelo del Suceso Empresarial (Shapero, 1982) y la Teoría de la Conducta Planificada (Ajzen, 1991), desde los que se asume que la intención de emprender deriva de variables de naturaleza cognitiva y media la influencia de éstas sobre la conducta de creación de empresas. A pesar de que los estudios llevados a cabo en la validación empírica de estos postulados han posibilitado un mejor conocimiento de la iniciativa emprendedora en la etapa universitaria, la literatura previa no ha sido capaz de probar el vínculo entre intenciones y conductas emprendedoras (Kolvereid, 1996a; Soutaris, Zerbinati y Al-Laham, 2007), al tiempo que existen diferencias entre autores en la conceptualización de las variables antecedentes de la intención (Chandler y Lyon, 2001; Liñán y Chen, 2009). En este contexto, algunos académicos han criticado el empleo de universitarios como muestras de conveniencia en la validación de la teoría general sobre creación de empresas (Hemmasi y Hoelscher, 2005; Muñoz Adánez, 1997, 1999; Robinson, Huefner, y Hunt, 1991).

Para superar tales limitaciones, este estudio incorpora los fundamentos teóricos de los modelos de intenciones emprendedoras en la literatura vocacional específicamente dedicada a explicar los procesos de elección de carrera en la juventud. Desde esta perspectiva, más que probar la validez de la teoría general sobre creación de empresas en estudiantes universitarios, se apuesta por una teoría de carrera para explicar la conducta emprendedora de los futuros graduados de acuerdo a las circunstancias académicas y profesionales características de dicha etapa. No en vano, el origen empresarial ha sido frecuentemente conceptualizado en términos de decisión de carrera entre las alternativas del empleo por cuenta propia y ajena (Bird, 1988; Douglas y Sheperd, 2002; Katz, 1992; Kolvereid, 1996a, 1996b; Soutaris et al., 2007). A tal respecto, la Teoría Social Cognitiva de Carrera (TSCC) de Lent, Brown y Hackett $(1994,2000)$ comparte con los modelos clásicos de intenciones emprendedoras la conceptualización del proceso de elección de carrera como una secuencia de intenciones y conductas, al tiempo que supone una menor dependencia del largo plazo en el estudio de las trayectorias académico profesionales tempranas y permite una mayor especificidad definicional de los precursores del desarrollo de carrera en términos de autoeficacia percibida y expectativas de resultados.

Profundizando en dichos argumentos, este trabajo pretende reconciliar la literatura previa sobre iniciativa emprendedora en la etapa universitaria mediante la propuesta de un modelo basado en los postulados teóricos de la TSCC. En la operativización de dicho propósito, se trata de dar respuesta a dos cuestiones: ¿puede explicarse el proceso de elección de carrera emprendedora de los jóvenes universitarios a partir de la relación entre intereses vocacionales, intenciones y conductas emprendedoras tempranas? y ¿están dichas variables relacionadas con la autoeficacia percibida y expectativas de resultados anticipadas en la creación de empresas?

\section{El estudio de la intención emprendedora en estu- diantes universitarios}

Las intenciones emprendedoras han sido definidas en alusión a los estados mentales que dirigen la atención, experiencia, y acción hacia un concepto de negocio (Bird, 1988), asumiéndose que la creación de una nueva empresa implica un plazo de tiempo más o menos largo de considerable planificación en la que el emprendedor no reacciona de forma automática ante los estímulos del medio, sino que procesa la información del ambiente que le rodea en un esfuerzo cognitivo de transformación de las oportunidades percibidas en forma de proposiciones de negocio viables (Katz y Gartner, 1988; Krueger, 1993). Desde este punto de vista, las intenciones emprendedoras son vistas como el primer paso del largo y complejo proceso de establecimiento y consolidación organizacional (Lee y Wong, 2004), concediéndose al estudio de sus desencadenantes una gran importancia en la comprensión del origen de la iniciativa emprendedora (Shane y Venkataraman, 2000; Shaver y Scott, 1991).

Los marcos teóricos más influyentes en la literatura en la explicación de la intencionalidad empresarial han sido el Modelo del Suceso Empresarial (Shapero, 1982) y la aplicación al campo de la Teoría de la Conducta Planificada (Ajzen, 1991). Ambos marcos teóricos consideran la intención de emprender como el principal desencadenante del comportamiento emprendedor subsiguiente, $\mathrm{y}$ argumentan que las intenciones pueden ser predichas a partir de variables de naturaleza actitudinal que influyen indirectamente sobre la conducta. La mayor parte de trabajos dedicados a la validación empírica de los modelos de intenciones emprendedoras han recurrido a muestras de estudiantes universitarios, con respaldo en el argumento de que, dada la sensibilidad de los procesos intencionales ante las condiciones iniciales que las elicitan (Kim y Hunter, 1993), la puesta a prueba del alcance de la teoría requiere estudiar el fenómeno emprendedor antes de que ocurra, en el contexto de la comparación entre emprendedores potenciales y otras personas sin intención de emprender (MacMillan y Katz, 1992). A tal respecto, el colectivo estudiantil es considerado representativo de individuos implicados en decisiones de carrera y con una amplia variedad de experiencias, intenciones y actitudes hacia la creación de empresas que les hacen especialmente idóneos para estos cometidos (Krueger, 1993; Krueger, Reilly y Carsrud, 2000). Desde este encuadre, los estudios basados en el Modelo del Suceso Empresarial prueban que la decisión de emprender de los estudiantes universitarios deriva en gran medida de las percepciones de deseabilidad y factibilidad asociadas a la creación de empresas (Fitzsimmons y Douglas, 2011; Krueger, 1993; Krueger et al., 2000), mien- 
tras que los trabajos fundamentados en la Teoría de la Conducta Planificada constatan la influencia directa sobre la intención de emprender de la actitud hacia la conducta y el control conductual percibido (Autio, Keeley, Klofsten, Parker y Hay, 2001; Kolvereid, 1996a; Krueger et al., 2000; Soutaris et al., 2007; Tkachev y Kolvereid, 1999) ${ }^{1}$. Los mismos resultados se han visto confirmados en varios estudios realizados a lo largo de la última década en universidades españolas (Díaz-Casero, Ferreira, Hernández-Mogollón y Raposo, 2012; Guerrero et al., 2008; Lanero, Vázquez, Gutiérrez y García, 2011; Liñán y Chen, 2009; Liñán, Urbano y Guerrero, 2011; Moriano, Gorgievski, Laguna, Stephan y Zarafshani, 2012; Toledano y Urbano, 2008; Veciana et al., 2005).

En la interpretación de los resultados mencionados, algunos autores han establecido correspondencias entre las variables consideradas por ambos modelos teóricos (Krueger, 1993, 2000; Krueger et al., 2000; Krueger y Brazeal, 1994; Krueger y Carsrud, 1993), pudiendo hablarse de dos grandes factores determinantes de la intención de emprender. De un lado, el constructo de factibilidad es equiparable al de control conductual percibido, representando la anticipación de la creación de una empresa como conducta realizable. De otro lado, la deseabilidad percibida es considerada equivalente a la actitud hacia la conducta, aludiendo a la percepción de la alternativa emprendedora como atractiva.

A pesar de los avances en la explicación de la intencionalidad empresarial en la etapa universitaria, la literatura previa adolece de dos limitaciones fundamentales, en torno a la relación lineal esperada entre intenciones y conductas, y a la definición de las actitudes consideradas precedentes de aquéllas.

En primer lugar, cabe apuntar la escasa utilidad de los modelos propuestos para predecir la conducta emprendedora real del alumnado, por cuanto que la aplicación de los modelos clásicos de intenciones emprendedoras en contextos universitarios ha estado más enfocada al análisis del vínculo actitudes-intención que a la forma en que dichas variables se traducen en la creación de nuevas empresas (Kolvereid, 1996a; Soutaris et al., 2007). Tal escasez de resultados encuentra fácilmente su explicación en el lapso temporal que a menudo separa el paso por la Universidad de la implicación en iniciativas empresariales, de modo que, independientemente de las preferencias e intenciones emprendedoras de los graduados, los estudios demuestran que el comienzo en los negocios se plantea a menudo como una meta a largo plazo (Collins, Hannon y Smith, 2004; Galloway, Anderson, Brown y Wilson, 2005; Johnson, Craig y Hildebrand, 2006; McLarty, 2005; Soutaris et al., 2007) que requiere de un cierto margen de tiempo para ganar experiencia y construir una oportunidad de negocio viable (Fiet y Pankaj, 2008), y que puede verse truncada por diversas circunstancias (Fayolle, 2005; Krueger y Carsrud, 1993; Shapero, 1982). A este res-

${ }^{1}$ Si bien la teoría de Ajzen (1991) defiende también la influencia de la norma subjetiva, dicha asociación no ha sido lo suficientemente constatada en la predicción de la intencionalidad empresarial de los estudiantes (Autio et al., 2001; Krueger et al., 2000; Liñán y Chen, 2009; Moriano et al., 2012). pecto, la dificultad técnica de llevar a cabo diseños longitudinales ha restringido el margen de maniobra de muchos investigadores al empleo de diseños transversales que no permiten dar cuenta de la relación causal entre intención de emprender y creación de una empresa. Con todo, diversos expertos son escépticos ante el empleo de universitarios como muestras de emprendedores potenciales en la validación de la teoría general sobre creación de empresas (Hemmasi y Hoelscher, 2005; Muñoz Adánez, 1997, 1999; Robinson et al., 1991).

En segundo lugar, en los estudios previos se aprecia una cierta diversidad en el contenido de las escalas utilizadas para predecir la intención de emprender, lo que dificulta la comparación y generalización de los resultados obtenidos (Chandler y Lyon, 2001; Liñán y Chen, 2009). De un lado, los constructos de factibilidad percibida y control conductual han sido estimados a través tanto de medidas generales en términos de dificultad, autoconfianza, control y certeza de éxito (Autio et al., 2001; Kolvereid, 1996a; Krueger, 1993; Liñán y Chen, 2009; Veciana et al., 2005), como de escalas de autoeficacia específicas del dominio (Krueger et al., 2000; Fitzsimmons y Douglas, 2011; Moriano, Gorgievski et al., 2012) Paralelamente, la equivalencia entre deseabilidad percibida y actitud hacia la conducta ha dado pie a hablar de dicha faceta cognitiva en términos de atracción general hacia la creación de empresas (Autio et al., 2001; Krueger, 1993; Liñán y Chen, 2009; Veciana et al., 2005), resultados esperados (Fitzsimmons y Douglas, 2011; Kolvereid, 1996a), o una combinación de ambas medidas (Krueger et al., 2000; Moriano, Gorgievski et al., 2012).

En tales circunstancias, vemos justificada la necesidad de avanzar hacia modelos prácticos que permitan identificar más operativamente las dimensiones individuales implicadas en el surgimiento de iniciativas emprendedoras en el colectivo universitario. En tal empeño, las teorías psicológicas desarrolladas específicamente para la explicación de la conducta de elección y desarrollo de carrera en el colectivo de adultos jóvenes pueden constituir una excelente alternativa a los modelos clásicos de intenciones emprendedoras, por su mayor proximidad a la realidad profesional de los futuros titulados superiores. En base a dicho argumento, revisamos a continuación los principales postulados de la teoría sobre desarrollo de carrera de Lent et al. $(1994,2000)$ y su aplicabilidad al estudio de la iniciativa emprendedora en universitarios.

\section{La Teoría Social Cognitiva de Carrera}

La TSCC de Lent et al. (1994, 2000) se ha convertido en una de las corrientes de referencia en la literatura sobre comportamiento vocacional de los últimos veinte años (Betz, 2008). En base a los principales postulados de la Teoría Social Cognitiva de Bandura (1977, 1986, 1997), el enfoque está centrado en la explicación de los mecanismos a través de los cuales los individuos ejercen control personal sobre los procesos comportamentales implicados en el desa- 
rrollo de carrera, perfilándose especialmente útil en la explicación de las fases iniciales de decisión y comportamiento vocacional de adolescentes y adultos jóvenes inmersos en su preparación para el acceso al mundo laboral. No en vano, los postulados de la TSCC han sido probados con éxito en muestras de estudiantes universitarios de diversos campos académicos (Blanco, 2009; Diegelman y Subich, 2001; Ferry, Fouad y Smith, 2000; Flores, Robitschek, Celebi, Andersen y Hoang, 2010; Gainor y Lent, 1998; Lent et al., 2001, 2003, 2005, 2008; Lent, López, López y Sheu, 2008; Lent, López, Sheu y López, 2011; Lent, Sheu, Gloster y Wilkins, 2010; Schaub y Tokar, 2005; Sheu et al., 2010; Tang, Fouad y Smith, 1999).

Desde este encuadre, la TSCC comparte con el Modelo del Suceso Empresarial (Shapero, 1982) y la Teoría de la Conducta Planificada (Ajzen, 1991) muchos de los postulados que defienden la secuencia entre variables de naturaleza cognitiva, intenciones y conductas como el núcleo central del desarrollo de carrera, al tiempo que ofrece la posibilidad de superar las principales limitaciones apuntadas para estos marcos teóricos en la explicación de la iniciativa emprendedora en la etapa universitaria. En primer lugar, Lent et al. (1994) conciben el desarrollo de metas de carrera como una secuencia de intereses, intenciones y conductas de elección ocupacional que implican una menor dependencia del largo plazo en el estudio del comportamiento emprendedor inmediato de los futuros graduados de acuerdo a sus aspiraciones vocacionales. En segundo lugar, la TSCC da pie a una mayor especificidad definicional de las variables precursoras del desarrollo de carrera en términos de autoeficacia y expectativas de resultados, atribuyendo a ambas múltiples influencias en la secuencia de metas de carrera en una visión más dinámica de los mecanismos psicológicos implicados en el comportamiento vocacional.

Profundizando en estos argumentos, las siguientes secciones revisan la fundamentación teórica de la TSCC y su aplicación a la explicación de la conducta emprendedora del colectivo universitario. Con respaldo en dicha revisión, la Figura 1 representa gráficamente las hipótesis de la investigación en el contexto de la contribución del marco teórico propuesto a los modelos previos de intenciones emprendedoras.

\section{Intereses, intenciones y conductas emprendedoras tempranas}

En línea con los modelos de intenciones emprendedoras, la TSCC concibe el proceso de elección de carrera como una secuencia entre intenciones y conductas vocacionales (Lent et al., 1994). Desde tal encuadre, las intenciones de carrera o metas de elección aluden a planes de dedicación a una determinada actividad. Por su parte, las conductas de elección de carrera son definidas como todas aquellas acciones específicas que marcan la entrada del individuo en una línea académica o profesional (i.e., conductas de búsqueda de empleo).
Asumiendo este enfoque, el resultado esperable a corto plazo de las intenciones emprendedoras de los estudiantes universitarios habría de ser conceptualizado en términos de conductas tempranas de iniciación empresarial como alternativa de carrera elegida, aún siendo previas a la creación final de una empresa. Desde esta aproximación, si bien no es frecuente que los recién titulados universitarios se embarquen en su propio negocio inmediatamente después de finalizar sus estudios, determinadas conductas aproximativas a dicha meta pueden ser observadas en jóvenes a punto de incorporarse al mundo laboral (Soutaris et al., 2007). De hecho, algunos programas prácticos de apoyo a la iniciativa emprendedora pioneros en las universidades de nuestro entorno dan cabida al propósito de que los estudiantes con más intención de emprender comiencen a realizar conductas aproximativas a tal fin (Placer y Vázquez, 2005; Ruíz et al., 2004).

Siguiendo dicha visión de la conducta emprendedora inicial, en la literatura especializada en creación de empresas se habla de un proceso de gestación de negocio o nascency que supone la implicación del individuo en actividades tempranas de puesta en marcha de una empresa, tales como la dedicación de tiempo y recursos personales, la elaboración de un plan de negocio, la participación en formación emprendedora o la búsqueda de recursos financieros (Aldrich y Martínez, 2001; Carter, Gartner y Reynolds, 1996; Reynolds y Miller, 1992). La mayor parte de estudios previos sobre el periodo de gestación previo a la creación de empresas han utilizado muestras extraídas de la población general de emprendedores potenciales (Alsos y Kolvereid, 1998; Arenius y Minniti, 2005; Davidsson y Honig, 2003; Hechavarría, Renko y Matthews, 2012; McGee, Mueller y Sequeira, 2009; Rotefos y Kolvereid, 2005; Sequeira, Mueller y McGee, 2007; Townsend, Busenitz y Arthurs, 2010), documentándose incluso una asociación positiva entre las intenciones y conductas emprendedoras tempranas (Kolvereid e Isaksen, 2006). No obstante, apenas existe evidencia sobre la conducta emprendedora inicial de los universitarios como respuesta inmediata a sus intenciones de carrera (Soutaris et al., 2007).

En respuesta a dicha escasez de resultados, y con respaldo en los postulados teóricos de la TSCC y de los modelos de referencia en la explicación de la intencionalidad emprendedora (Ajzen, 1991; Shapero, 1982), proponemos la siguiente hipótesis:

Hipótesis 1: La intención emprendedora tiene un efecto positivo directo sobre la conducta emprendedora temprana.

A su vez, la TSCC defiende que las intenciones de carrera derivan de la formación de intereses vocacionales y median la influencia de éstos sobre las conductas de elección finalmente implementadas (Lent et al., 1994). Desde este encuadre, los intereses son definidos como patrones de atracción temprana hacia las actividades relacionadas con una ocupación (Hansen, 1984).

Frente a los modelos clásicos de intenciones, la consideración de los intereses profesionales como variables precursoras del proceso de elección de carrera posibilita una explicación de la iniciativa emprendedora más próxima a la reali- 
dad concreta que marca la configuración de trayectorias académico-profesionales iniciales en la juventud. No obstante, el constructo de intereses emprendedores y su impacto en la construcción de proyecciones laborales acordes ha sido estudiado especialmente en la etapa de la adolescencia (Bonnett y Furnham, 1991; Schmitt-Rodermund, 2004; SchmittRodermund y Vondracek, 2002, Schröder y SchmittRodermund, 2006), por considerarse éste el momento en que los intereses profesionales comienzan a consolidarse. En cambio, la evolución de dichas aspiraciones iniciales en etapas posteriores y más próximas a la transición al mundo laboral resulta desconocida.

Es por ello por cuanto la principal evidencia acerca de los intereses de los jóvenes con estudios superiores por el desempeño de funciones laborales típicamente empresariales remite a la tipología RIASEC ${ }^{2}$ de intereses vocacionales de Holland (1959, 1985, 1997), dentro de la cual la categoría Empresarial define la preferencia por actividades ocupacionales relativas al mundo de la gestión organizacional (Flores et al., 2010; Schaub y Tokar, 2005; Sheu et al., 2010). Los mismos trabajos han evidenciado el efecto directo de los intereses sobre las intenciones ocupacionales.

Si bien tal línea de trabajo supone una primera aproximación a los intereses empresariales de los universitarios, la iniciativa emprendedora requiere, por lo general, de habilidades más complejas de las requeridas para la organización y gestión empresarial (Gibb, 1983; Hynes, 1996; Levenburg, Lane, Schwarz y Rapids, 2006; McMullan y Long, 1987; Sexton y Bowman, 1984), precisándose de estudios focalizados en el refinamiento teórico y metodológico del constructo. En base a dicho propósito y de acuerdo a los postulados de Lent et al. (1994), en este trabajo proponemos las siguientes hipótesis:

Hipótesis 2: Los intereses emprendedores tienen un efecto positivo directo sobre la intención emprendedora.

Hipótesis 3: Los intereses emprendedores tienen un efecto positivo indirecto sobre la conducta emprendedora temprana, por mediación de la intención emprendedora.

\section{Autoeficacia emprendedora y expectativas de resul- tados}

La TSCC propone la autoeficacia y las expectativas de resultados como los principales precursores del desarrollo de carrera. Desde este encuadre, la autoeficacia percibida se refiere a las creencias de capacidad para llevar a cabo las conductas implicadas en la consecución de determinadas metas académicas o profesionales (Lent y Brown, 2006). Asimismo, las expectativas de resultados aluden a las creencias personales sobre las consecuencias de realizar una determinada

${ }^{2}$ El modelo RIASEC de Holland $(1959,1985,1997)$ representa una de las teorías más influyentes en la literatura vocacional sobre formación y desarrollo de intereses de carrera. El acrónimo que da nombre a la propuesta toma como referencia las iniciales de las seis categorías de ocupaciones identificadas por el autor: Realista, Investigador, Artístico, Social, Empresarial, y Convencional. conducta (Lent et al., 1994). En base a estas definiciones, la TSCC comparte con los modelos de intenciones emprendedoras la premisa básica de que las personas desarrollan sus aspiraciones profesionales en el ámbito de las actividades que perciben realizables y potencialmente beneficiosas (Bandura, 1986; Lent et al., 1994).

En el campo que nos ocupa, la autoeficacia emprendedora ha sido definida como la confianza de la persona en su capacidad para llevar a cabo las tareas necesarias para crear una empresa (Chen, Greene y Crick, 1998). Si bien algunos autores han considerado el constructo equivalente a los de factibilidad percibida y control conductual en la predicción de la intencionalidad emprendedora (Fitzsimmons y Douglas, 2011; Krueger, 2000; Krueger et al., 2000; Moriano, Gorgievski et al., 2012), ciertos matices sugieren que la autoeficacia alude a un concepto de mayor especificidad conductual que los otros dos. Así, Ajzen (2002) ha afirmado que el control conductual percibido engloba un doble componente de competencia personal y de controlabilidad -relativa al grado en que el rendimiento depende del actor-, concepto este último que se escapa a la definición de autoeficacia (Bandura, 1977, 1986, 1997). Asimismo, diversos autores abogan por el empleo de medidas de autoeficacia emprendedora específicas de dominio (Chen et al., 1998; De Noble, Jung y Ehrlich, 1999; McGee et al., 2009), frente a otras medidas generales de factibilidad conductual en términos de dificultad, autoconfianza y certeza de éxito (Autio et al., 2001; Kolvereid, 1996a; Krueger, 1993; Liñán y Chen, 2009; Veciana et al., 2005).

En la misma línea, el concepto de expectativas de resultados relativo a la anticipación de consecuencias favorables en la creación de empresas ha estado presente en buena parte de la literatura sobre intenciones emprendedoras, definiendo la deseabilidad o actitud personal hacia la conducta (Fitzsimmons y Douglas, 2011; Kolvereid, 1996a; Krueger et al., 2000; Moriano, Gorgievski et al., 2012). No obstante, desde un marco de expectativa-valor, se asume que las expectativas de resultados determinan la formación de actitudes positivas cuando dichas consecuencias son valoradas (Ajzen, 2001), entendiéndose que, aunque ambas variables están relacionadas, representan constructos independientes. En la misma línea, algunos autores diferencian entre la anticipación de resultados favorables en la creación de empresas y la valoración afectiva de dicha conducta (Liñán y Chen, 2009; Goethner, Obschonka, Silvereisen y Cantner, 2012). Dicha puntualización sugiere la pertinencia de diferenciar las expectativas de resultados emprendedores de otras mediciones afines de atracción personal y evaluación general positiva del autoempleo en la predicción de las intenciones y conductas emprendedoras (Autio et al., 2001; Krueger, 1993; Krueger et al., 2000; Liñán y Chen, 2009; Moriano, Gorgievski et al., 2012).

Asimismo, la TSCC propone que las expectativas de resultados están parcialmente determinadas por las percepciones de autoeficacia, por cuanto que generalmente las personas esperan resultados deseables en actividades para las que 
se sienten competentes (Bandura, 1986, 1997). Paralelamente, diversos estudiosos han argumentado que personas con distinto nivel de competencia subjetiva para emprender podrían anticipar distintos tipos de resultados en la iniciativa empresarial (Boyd y Vozikis, 1994; Chen et al., 1998; De Noble et al., 1999). Con todo, proponemos la siguiente hipótesis:

Hipótesis 4: La autoeficacia emprendedora tiene un efecto positivo directo sobre las expectativas de resultados.

Más allá de las especificidades definicionales comentadas, la TSCC también aporta a los modelos previos de intenciones emprendedoras una visión más dinámica de los efectos de la autoeficacia y las expectativas de resultados en la secuencia de metas de carrera, hablando de una doble vía de influencia directa sobre los intereses, intenciones y conductas emprendedoras tempranas, e indirecta por mediación de las variables sociocognitivas precedentes en el modelo.

Comenzando por la influencia más remota de la autoeficacia en la configuración de metas emprendedoras, los escasos estudios disponibles en adolescentes y estudiantes universitarios avalan la relación entre competencia percibida e intereses vocacionales por el desarrollo de carreras vinculadas al mundo empresarial (Flores et al., 2010; Schaub y Tokar, 2005; Schmitt-Rodermund y Vondracek, 2002; Sheu et al., 2010), al tiempo que se ha probado el efecto positivo de la autoeficacia sobre la intención emprendedora de estudiantes universitarios (Chen et al., 1998; De Noble et al., 1999; Kickul, Gundry, Barbosa y Whitcanack, 2009; Moriano, Palací y Morales, 2006; Moriano, Topa, Molero, Entenza y Lévy-Mangin, 2012; Wilson, Kickul y Marlino, 2007; Zhao, Seibert y Hills, 2005; Zellweger, Sieger y Halter, 2011). Asimismo, existe sobrada evidencia sobre la relación entre autoeficacia y conducta empresarial subsiguiente (Arenius y Minniti, 2005; Chen et al., 1998; Hechavarría et al., 2012; Koellinger, Minniti y Schade, 2007; Markman, Balkin y Baron, 2002; Markman, Baron y Balkin, 2005; McGee et al., 2009; Sequeira et al., 2007; Townsend et al., 2010), si bien este vínculo no ha sido analizado en muestras de universitarios para explicar su conducta emprendedora temprana. En un esfuerzo integrador de tales resultados con los postulados de la TSCC, planteamos las siguientes hipótesis:

Hipótesis 5: La autoeficacia emprendedora tiene un efecto positivo sobre los intereses emprendedores, tanto directo como indirecto por mediación de las expectativas de resultados.

Hipótesis 6: La autoeficacia emprendedora tiene un efecto positivo sobre la intención emprendedora, tanto directo como indirecto por mediación de las expectativas de resultados y los intereses emprendedores.

Hipótesis 7: La autoeficacia emprendedora tiene un efecto positivo sobre la conducta emprendedora temprana, tanto directo como indirecto por mediación de las expectativas de resultados, los intereses y la intención emprendedora.

Por lo que respecta a las expectativas de resultados, algunas evidencias apoyan igualmente el efecto de dicha variable sobre la formación de intereses empresariales en la ju- ventud (Schaub y Tokar, 2005; Sheu et al., 2010). Asimismo, diversos estudios se han ocupado de la relación entre anticipación de recompensas e intenciones y conductas emprendedoras a través del análisis de las razones que llevan a las personas a emprender. Desde este enfoque, se asume un concepto de expectativas de resultados basado en el valor de determinadas consecuencias para el emprendedor potencial, tratando de demostrarse el vínculo entre ciertas motivaciones -como la búsqueda de independencia, el desarrollo personal o la consecución de rentabilidad económica- y la elección de carreras emprendedoras. Específicamente, algunos estudios han tomado mediciones de la importancia asignada a las consecuencias esperables en la creación de empresas para predecir las intenciones de estudiantes universitarios (Brenner, Pringle y Greenhaus, 1991; Douglas y Sheperd, 2002; Fitzsimmons y Douglas, 2011; Kolvereid, 1996a, 1996b; Krueger et al., 2000; Moriano, Gorgievski et al., 2012) o estudiar la conducta inicial de emprendedores nacientes (Carter, Gartner, Shaver y Gatewood, 2003; Cassar, 2007; Edelman, Brush, Manolova y Greene, 2010; Gatewood, Shaver y Gartner, 1995; Renko, Kroeck y Bullough, 2012).

Si bien los trabajos citados han resultado eficaces a la hora de probar el vínculo entre consecuencias esperadas y elección de carreras emprendedoras, lo cierto es que se aprecia también una escasa integración teórica en la operativización de los motivos específicos que llevan a emprender. En relación a ello, Carter et al. (2003) advierten de que es difícil proponer modelos generalizables sobre la motivación emprendedora, dado que los resultados pueden estar sesgados por el momento temporal o las circunstancias socioeconómicas imperantes en el país de cada estudio. Asimismo, los autores argumentan que en estudios prospectivos en los que se pregunta de manera previa a la implementación de la elección de carrera, las razones mencionadas por los emprendedores no difieren sustancialmente de las reportadas para otras alternativas. En línea con dicho argumento, la TSCC asume un concepto de expectativas de resultados basado en la instrumentalidad o utilidad atribuida a una carrera para conseguir determinadas recompensas (Lent et al., 1994). Desde este encuadre, más que analizar los motivos vinculados a una determinada elección de carrera, los trabajos empíricos desarrollados en esta línea buscan cuantificar la magnitud de las recompensas anticipadas para predecir la predisposición de carrera (Blanco, 2009; Lent et al., 2001, 2003, 2005, 2008).

Uno de los pocos estudios que ha asumido esta perspectiva en la literatura previa ha sido el de Mitchell y Knudsen (1973), quienes diferenciaron entre expectativas de resultados extrínsecos e intrínsecos en la creación de empresas en función, respectivamente, de si la conducta emprendedora estuviese motivada por la anticipación de resultados directamente derivados de su ejecución (e.g., salario, promoción, seguridad, estatus, etc.) o por el propio interés y satisfacción inherentes a la misma (e.g., creatividad, interacción social, desarrollo personal, etc.). Muchos de los trabajos menciona- 
dos y conducidos más recientemente en el análisis de las razones de carrera vinculadas a la iniciativa empresarial (Carter et al., 2003; Kolvereid, 1996b) han dado cabida a ambos tipos de motivadores, encontrándose que las expectativas de resultados de ambas categorías son importantes en la predicción de la intención y conducta emprendedoras. Con el propósito de clarificar dicho patrón de resultados al amparo de las predicciones de la TSCC planteamos las siguientes hipótesis:
Hipótesis 8: Las expectativas de resultados tienen un efecto positivo directo sobre los intereses emprendedores.

Hipótesis 9. Las expectativas de resultados tienen un efecto positivo sobre la intención emprendedora, directo e indirecto por mediación de los intereses emprendedores.

Hipótesis 10: Las expectativas de resultados tienen un efecto positivo sobre la conducta emprendedora temprana, directo e indirecto por mediación de los intereses y la intención emprendedora.

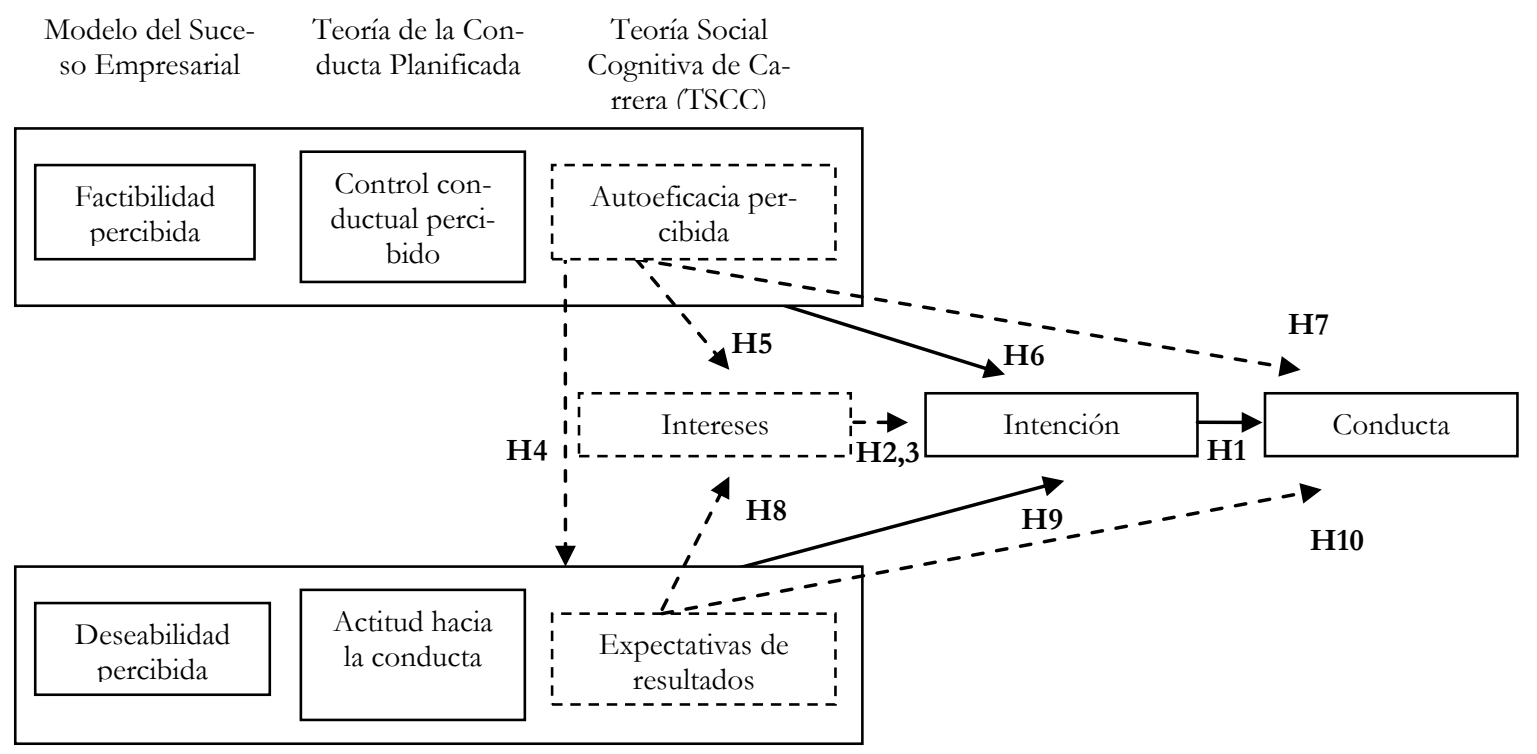

Figura 1. Modelo de hipótesis.

Nota. Las líneas discontinuas identifican la contribución de la TSCC a los modelos de intenciones emprendedoras

\section{Método}

\section{Participantes}

Los datos del estudio fueron recogidos en el periodo de Febrero a Junio de 2010 a partir de la encuesta a una muestra representativa de 400 estudiantes de la Universidad Complutense de Madrid. La población objeto de estudio quedó definida en base al total de matriculados en las titulaciones en extinción de Primer y Segundo Ciclo impartidas en dicha universidad durante el periodo académico 2009/2010, bajo la pretensión de basar las conclusiones del trabajo en la experiencia de partícipes de enseñanzas superiores regladas de larga tradición ${ }^{3}$.

En términos más específicos, la composición de la muestra derivó de un procedimiento de muestreo estratificado, garantizándose la representatividad de los estudiantes de acuerdo a la clásica distinción por áreas académicas. Siguiendo este criterio, el $61.2 \%$ de los encuestados cursaba titulaciones de Ciencias Sociales y Jurídicas, el 12.8\% de Humani-

\footnotetext{
${ }^{3}$ Los nuevos Títulos de Grado comenzaron a implantarse en algunas universidades españolas durante los cursos académicos 2008-09 y 2009-10, llegando a verificarse por entonces un total de 1.275 Grados, lo que supone un
} $47.8 \%$ del proceso de adaptación a completar para el curso 2010-11. dades, el 11.1\% de Ciencias de la Salud, el 10.7\% de Ciencias Experimentales, y el $4.2 \%$ de Enseñanzas Técnicas.

Cabe precisar asimismo que, con el propósito de garantizar la máxima experiencia universitaria posible de los participantes en la investigación, se trató de concentrar la recogida de información en los últimos cursos de carrera. En este sentido, el $77.5 \%$ de alumnos encuestados estaba matriculado en los cursos cuarto y quinto de Licenciatura o Ingeniería, correspondiendo el $22.55 \%$ restante a alumnado de tercer curso, predominantemente de Diplomaturas o Ingenierías Técnicas.

Por lo que respecta a las características sociodemográficas de los encuestados, la muestra estuvo integrada por 307 mujeres $(76.8 \%)$ y 93 hombres $(23.3 \%)$, con edades comprendidas entre los 20 y los 29 años, siendo la media de 23.09 años $(D T=3.03)$.

\section{Procedimiento}

La recogida de datos para el estudio estuvo centrada en la administración de un cuestionario a la muestra selecciona$\mathrm{da}$, el cual fue construido en su mayor parte para los propósitos de la investigación.

Para garantizar la validez de contenido, el desarrollo de los ítems a integrar en las escalas de medición siguió una 
aproximación deductiva (Hinkin, 1995; Martínez, 1995; Schwab, 1980), basada en la definición operativa de los constructos teóricos a partir de una profunda revisión de la literatura especializada en el tema y de otros instrumentos similares. Asimismo, se trató de reducir al máximo la ocurrencia de errores de medida sistemáticos asociados al método psicométrico cuando las variables objeto de estudio deben ser obtenidas de la misma fuente y no pueden ser medidas en distintos contextos temporales o espaciales (Bagozzi y Yi, 1991; Fiske, 1982; Podsakoff, MacKenzie, Lee y Podsakoff, 2003).

La versión final del cuestionario quedó supeditada a un cuidadoso refinamiento de los ítems incluidos en base a la opinión de tres expertos en el área acerca de la adecuación de los indicadores propuestos en la medición de las variables de interés, así como a una prueba pre-test realizada con un reducido grupo de estudiantes universitarios a quienes se consultó acerca de la dificultad y comprensión del contenido de las escalas.

Una vez configurada la versión definitiva del cuestionario, se establecieron contactos personales por e-mail con diversos profesores responsables de asignaturas impartidas en los cursos y áreas de conocimiento objetivo. Por esta vía, los profesores eran informados sobre los propósitos y detalles de la investigación y se les solicitaba permiso para administrar el cuestionario en alguna de sus clases. En caso de obtener respuesta afirmativa de colaboración, un miembro del equipo de investigación se desplazaba a cada aula en la fecha y hora consensuadas para informar a los estudiantes asistentes sobre los objetivos de la investigación y requerir su participación voluntaria y anónima. A continuación, se procedía al reparto del cuestionario entre los voluntarios para su autoadministración colectiva en soporte de lápiz y papel, siendo el tiempo medio de respuesta de aproximadamente 20 minutos. En todo momento, el investigador permanecía presente a disposición de cualquier duda o aclaración que pudiese surgir.

Los cuestionarios reunidos eran revisados previamente a su codificación, eliminándose todos aquéllos que presentaban respuestas incompletas o sospechosas en base a patrones de tendencia de respuesta. Con todo, el mismo procedimiento fue repetido en diversas aplicaciones colectivas hasta completar el tamaño requerido de la muestra para cada rama académica.

\section{Instrumentos}

Intención emprendedora. La intención de emprender fue estudiada a través de la adaptación de una medida compuesta de tres ítems desarrollada inicialmente por Kolvereid (1996a) y adoptada posteriormente por otros autores (Soutaris et al., 2007; Tkachev y Kolvereid, 1999). Desde dicho planteamiento, la intencionalidad emprendedora fue entendida en términos de preferencia laboral por el autoempleo frente al trabajo por cuenta ajena y probabilidad anticipada de la decisión entre ambas alternativas. En cada caso, los encuestados debían valorar su grado de identificación con el enunciado de los ítems, de acuerdo a una escala de 0 (nada identificado) a 10 (completamente identificado).

Conducta emprendedora temprana. Con respaldo en la literatura previa (e.g., Alsos y Kolvereid, 1998; Carter et al., 2003; McGee et al., 2009; Rotefos y Kolvereid, 2005; Sequeira et al., 2007; Soutaris et al., 2007), la conducta emprendedora temprana fue estimada a partir de la identificación de los encuestados con cuatro enunciados referidos al grado de implicación en actividades preparatorias para la creación de empresas (e.g., "estoy reuniendo recursos para crear una empresa"). Las respuestas fueron clasificadas de acuerdo a una escala Likert de 0 (nada identificado) a 10 (completamente identificado).

Intereses emprendedores. Siguiendo el procedimiento típicamente empleado en estudios previos sobre la formación de intereses emprendedores durante la adolescencia (SchmittRodermund, 2004; Schmitt-Rodermund y Vondracek, 2002; Schröder y Schmitt-Rodermund, 2006), los participantes en el estudio fueron instados a manifestar el grado en que se sentían atraídos vocacionalmente por el desarrollo de 11 actividades profesionales relacionadas con la iniciativa emprendedora, de acuerdo a una escala de 0 (no me suscita ningún interés) a 10 (me suscita máximo interés). La especificación del contenido de los ítems estuvo basada en un trabajo de Liao, Armstrong y Rounds (2008), quienes han validado un largo listado de indicadores propuestos como referencia para el diseño de instrumentos de medición de los intereses vocacionales en distintos dominios, incluyendo algunos vinculados a la conducta emprendedora, como el mundo de los negocios (e.g., "idear formas de cubrir las necesidades de los consumidores"), las finanzas (e.g., "aprender principios básicos de Economía”), la gestión de recursos humanos (e.g., "organizar y supervisar la mano de obra de una empresa") y la gestión empresarial (e.g., "comprender cómo se gestiona la economía de una empresa”). Desde este encuadre, los ítems fueron seleccionados y adaptados en base a su coherencia con la especificidad del dominio emprendedor utilizado en la medición del resto de variables (i.e., la autoeficacia emprendedora).

Autoeficacia emprendedora. La dimensión de autoeficacia emprendedora fue estimada a partir de diez ítems relativos al sentido de competencia para el desempeño de actividades relacionadas con la creación y gestión de empresas, de acuerdo al planteamiento asumido en investigaciones previas sobre el constructo entendido como específico de dominio (e.g., Chen et al., 1998; De Noble et al., 1999; McGee et al., 2009; Zhao et al., 2005). Más operativamente, la selección de las actividades emprendedoras en base a las que construir el enunciado de los ítems estuvo basada en el modelo de autoeficacia para la creación de empresas de McGee et al. (2009), en torno a las fases de búsqueda de recursos (e.g., "identificar una oportunidad de negocio en el mercado"), planificación (e.g., "planificar las distintas áreas de funcionamiento de una empresa"), reunión de recursos (e.g., "reunir los recursos necesarios para crear una empresa"), e implementación (e.g., 
"dirigir y gestionar el funcionamiento de una empresa"). Para cada ítems, los encuestados debían indicar su grado de confianza en sí mismos para realizar las actividades listadas, de acuerdo a una escala tipo Likert de 0 (ninguna confianza) 10 (máxima confianza).

Expectativas de resultados. Siguiendo el procedimiento utilizado por otros estudios de elección de carrera basados en la TSCC (e.g., Blanco, 2009; Lent et al., 2001, 2003, 2005, 2008), las expectativas de resultados fueron estimadas a partir del grado de acuerdo reportado por los encuestados con respecto a la obtención de 14 potenciales consecuencias favorables a través de la creación de una empresa, utilizando una escala Likert de 0 (completamente en desacuerdo) a 10 (completamente de acuerdo). Desde este encuadre, los enunciados fueron adaptados de investigaciones previas sobre motivación emprendedora (e.g., Brenner et al., 1991; Carter et al., 2003; Douglas y Sheperd, 2002; Fitzsimmons y Douglas, 2011; Gatewood et al., 1995; Kolvereid, 1996a, 1996b; Krueger et al., 2000; Mitchell y Knudsen, 1973; Moriano, Gorgievski et al., 2012; Renko et al., 2012; Soutaris et al., 2007). Con el fin de comprobar la posible multidimensionalidad del constructo (e.g., Mitchell y Knudsen, 1973) y su funcionamiento diferencial en el modelo, se llevó a cabo un análisis factorial exploratorio con el programa SPSS $15.0^{4}$, que permitió la descomposición de la escala original en dos factores explicativos del $68.57 \%$ de la varianza total. El primero de ellos integró seis ítems relativos a la anticipación de resultados extrínsecos en la creación de empresas (e.g., "retribución económica"). El segundo factor integró ocho enunciados relativos a la anticipación de resultados intrínsecos (e.g., "autonomía e independencia en el trabajo"). Ambos constructos fueron tratados como constructos diferentes en la puesta a prueba del modelo empírico (Figura 2).

Información sociodemográfica. Con fines descriptivos de la muestra, un último apartado del cuestionario estuvo dedicado a recabar información personal sobre los estudios cursados, el sexo y la edad de los encuestados.

\section{Análisis de datos}

El tratamiento estadístico de la información recabada estuvo dirigido a analizar la fiabilidad y validez de las medidas incluidas en el cuestionario, y el cumplimiento de las hipótesis de la investigación. A tales propósitos, se empleó una técnica de modelos de ecuaciones estructurales basada en el método de mínimos cuadrados parciales (en inglés, partial least squares -PLS-), a través del programa estadístico SmartPLS 2.0 (Ringle, Wende y Will, 2005).

Mientras los métodos de modelado estructural basados en el análisis de la covarianza persiguen la estimación de parámetros y medidas de ajuste global del modelo, la técnica PLS tiene como objetivo maximizar la varianza explicada por las variables observadas y latentes, mediante la estimación de mínimos cuadrados ordinarios (ordinary least squares -

\footnotetext{
${ }^{4}$ Resultados disponibles por petición a los autores.
}

$\left.O L S_{-}\right)$y análisis de componentes principales. En este contexto, el tratamiento estadístico de los datos corresponde a lo que se conoce como una "modelización flexible" (Wold, 1980) basada en la creación de relaciones predictivas lineales óptimas entre variables sin realizar suposiciones a niveles de medida, distribuciones de los datos o tamaño muestral (Chin, 1998a; Fornell y Bookstein, 1982; Wold, 1985). Con todo, en comparación con los métodos basados en la covarianza, PLS se adapta mejor a aplicaciones exploratorias y de desarrollo de la teoría (Barclay, Higgins y Thompson, 1995; Chin, Marcolin y Newsted, 2003), tal es el caso de la presente investigación orientada a proponer un modelo alternativo a la explicación de la iniciativa emprendedora en poblaciones universitarias a partir de la TSCC.

El procedimiento PLS permite estimar el modelo de investigación en dos etapas. La primera de ellas implica evaluar la fortaleza del modelo de medida (u outer mode) en términos de fiabilidad individual de los ítems, consistencia interna o fiabilidad de las escalas y validez de constructo convergente y divergente. Una vez verificada la fiabilidad y validez de los instrumentos de medida, la segunda etapa se centra en la estimación de los parámetros de ajuste para el modelo estructural (o inner moded), informando sobre el cumplimiento de las hipótesis de investigación a través de coeficientes de regresión estandarizados (paths) e índices $R^{2}$.

\section{Resultados}

La Tabla 1 resume los resultados obtenidos en la validación del modelo de medida utilizado en la investigación a través del procedimiento PLS.

La fiabilidad individual de los ítems fue valorada examinando la significación de las cargas factoriales estandarizadas ( $\lambda$ ) o correlaciones simples de los indicadores con sus respectivos constructos. Los resultados iniciales mostraron que un indicador de la intención emprendedora presentaba una baja carga en el constructo $(<.50)$, por lo que fue eliminado de la estructura factorial. En un nuevo análisis, todas las cargas factoriales superaron el mínimo recomendado de .50 (Barclay et al., 1995; Chin 1998a, 1998b), de acuerdo a un nivel de significación de $p<.05$ calculado mediante un procedimiento bootstrap con 500 submuestras de 100 casos. Consecuentemente, los valores de comunalidad $\left(\lambda^{2}\right)$ de cada indicador se situaron por encima de .25 (Bollen, 1989), infiriéndose que los constructos latentes explicaron entre el 45\% y el $83 \%$ de la varianza en sus respectivos indicadores.

Para la medición de la consistencia interna de las escalas, el programa SmartPLS arroja dos indicadores: el tradicional alpha de Cronbach $(\alpha)$ y la fiabilidad compuesta del contructo $\left(\varrho_{c}\right)$, ésta última considerada por algunos autores como una medida superior a la otra por su independencia del número de atributos asociados a cada concepto (Fornell y Lacker, 1981). La interpretación de ambos índices es bastante similar, considerándose apropiados valores por encima de .70 (Barclay et al., 1995; Hair, Anderson, Tatham y Black, 1998; Nunnally, 1978; Nunnally y Bernstein, 1994). En tér- 
minos generales, los resultados obtenidos en este caso evidenciaron el cumplimiento de tal requisito, asegurando la minimización del error de medida en los indicadores utilizados. Únicamente los dos ítems finalmente empleados en la medición de la intención de emprender se asociaron a un alpha de Cronbach de .67. No obstante, dado que el índice de fiabilidad compuesta arrojó un resultado considerablemente más robusto, decidió conservarse la medida como tal en los análisis subsiguientes.

Tabla 1. Fiabilidad y validez del modelo de medida.

\begin{tabular}{|c|c|c|c|c|c|}
\hline Item/Constructo & $\lambda$ & $\lambda^{2}$ & $\alpha$ & $\varrho_{c}$ & AVE \\
\hline Intención emprendedora & & & .67 & .85 & .74 \\
\hline Preferencia por la creación de empresas & $.81 * * *$ & 66 & & & \\
\hline Probabilidad de crear una empresa & $.91 * * *$ & .83 & & & \\
\hline Conducta emprendedora temprana & & & .86 & 91 & .71 \\
\hline Asistencia a cursos/conferencias & $.75^{* * *}$ & .56 & & & \\
\hline Desarrollo de una idea de negocio & $.85^{* * *}$ & .72 & & & \\
\hline Reunión de recursos & $.90 * * *$ & 81 & & & \\
\hline Actividades de puesta en marcha & $.87 * * *$ & .76 & & & \\
\hline Intereses emprendedores & & & .94 & .95 & 61 \\
\hline Aprender principios básicos de Economía & $.67 * * *$ & .45 & & & \\
\hline Idear formas de cubrir necesidades & $.71 * * *$ & .50 & & & \\
\hline Planificar sistemas de negocio & $.85^{* * *}$ & .72 & & & \\
\hline Elaborar productos/servicios para el mercado & $.76^{* * *}$ & .58 & & & \\
\hline Vender productos/servicios a los consumidores & $.77 * * *$ & .59 & & & \\
\hline Desarrollar estrategias de publicidad & $.77 * * *$ & .59 & & & \\
\hline Mantener rels. con proveedores/distribuidores & $.85^{* * *}$ & .73 & & & \\
\hline Mantener relaciones con inversores & $.84 * * *$ & .70 & & & \\
\hline Comprender temas de gestión empresarial & $.80^{* * * *}$ & 64 & & & \\
\hline Organizar y supervisar recursos humanos & $.81 * * *$ & 65 & & & \\
\hline Aprender a dirigir una empresa & $.76^{* * *}$ & .58 & & & \\
\hline Autoeficacia emprendedora & & & .94 & 95 & .66 \\
\hline Identificar una oportunidad de negocio & $.78^{* * *}$ & .61 & & & \\
\hline Desarrollar un proyecto empresarial viable & $.84^{* * *}$ & .71 & & & \\
\hline Planificar áreas de funcionamiento empresarial & $.86^{* * *}$ & .74 & & & \\
\hline Reunir recursos para crear una empresa & $.79 * * *$ & .62 & & & \\
\hline Organizar recursos para crear una empresa & $.86^{* * *}$ & .74 & & & \\
\hline Dirigir y gestionar una empresa & $.88^{* * *}$ & .77 & & & \\
\hline Organizar operaciones de producción/ logística & $.86^{* * * *}$ & .74 & & & \\
\hline Comercializar productos y servicios & $.79 * * *$ & 62 & & & \\
\hline Reclutar y dirigir empleados & $.74 * * *$ & .55 & & & \\
\hline Llevar la contabilidad y administración & $.72 * * *$ & .52 & & & \\
\hline Expectativas de resultados extrinsecos & & & 91 & .93 & .69 \\
\hline Retribución económica & $.86^{* * * *}$ & .73 & & & \\
\hline Seguridad/estabilidad laboral & $.84 * * *$ & .70 & & & \\
\hline Conciliación personal y laboral & $.75^{* * * *}$ & .56 & & & \\
\hline Estatus social & $.86^{* * *}$ & .73 & & & \\
\hline Promoción profesional & $.87 * * *$ & .76 & & & \\
\hline Prestigio/reconocimiento social & $.80^{* * *}$ & .64 & & & \\
\hline Expectativas de resultados intrinsecos & & & .93 & 94 & .67 \\
\hline Autonomía/independencia laboral & $.70^{* * *}$ & .49 & & & \\
\hline Autorrealización personal & $.84^{* * *}$ & .70 & & & \\
\hline Trabajo interesante & $.86^{* * *}$ & .73 & & & \\
\hline Variedad de tareas laborales & $.81^{* * *}$ & .66 & & & \\
\hline Oportunidades de aprendizaje & $.84 * * *$ & .70 & & & \\
\hline Ajuste habilidades-exigencias laborales & $.84 * * *$ & .70 & & & \\
\hline Satisfacción profesional & $.86^{* * *}$ & .74 & & & \\
\hline Autoestima/imagen positiva de uno mismo & $.82 * * *$ & .67 & & & \\
\hline
\end{tabular}

Autoestima/imagen positiva de uno mismo

${ }^{*} p<.001$ ( $t$ de Student con 499 grados de libertad).

La validez convergente fue estimada a partir de la medida de la varianza extraída media (Average variance extracted $A V E-)$, que determina la cantidad de varianza que un constructo obtiene de sus indicadores con relación a la cantidad de varianza debida al error de medida. Los valores $A V E$ asociados a todas las variables latentes superaron el mínimo recomendado de .50 (Fornell y Lacker, 1981). Asimismo, la validez discriminante fue evaluada a partir de la verificación 
de que cada constructo compartiera más varianza con sus medidas o indicadores que con otros constructos del modelo (Barclay et al., 1995). Para comprobar tal condición, la Tabla 2 muestra la matriz de correlaciones entre las variables laten- tes, reemplazando la diagonal por la raíz cuadrada de los valores $A V E$, los cuales fueron superiores al resto de índices $r$ (Fornell y Lacker, 1981).

Tabla 2. Matriz de correlaciones y raíz cuadrada de $A V E$ (diagonal).

\begin{tabular}{|c|c|c|c|c|c|c|c|c|}
\hline & $M$ & $D T$ & 1 & 2 & 3 & 4 & 5 & 6 \\
\hline 1. Intención emprendedora & 4.02 & 2.47 & $(.86)$ & & & & & \\
\hline 2. Conducta emprendedora temprana & 1.05 & 1.66 & .50 & $(.84)$ & & & & \\
\hline 3. Intereses emprendedores & 5.35 & 2.33 & .47 & .26 & $(.78)$ & & & \\
\hline 4. Autoeficacia emprendedora & 4.82 & 2.14 & .51 & .42 & .48 & $(.81)$ & & \\
\hline 5. Expect. de resultados extrínsecos & 6.71 & 2.06 & .29 & .08 & .36 & .31 & $(.83)$ & \\
\hline 6. Expect. de resultados intrínsecos & 7.76 & 1.75 & .28 & .04 & .44 & .25 & .67 & $(.82)$ \\
\hline
\end{tabular}

$M$ (media); DT (desviación típica).

Una vez comprobada la fiabilidad y validez del modelo de medida, se utilizó la técnica PLS para poner a prueba las relaciones hipotetizadas entre variables latentes exógenas y endógenas. La significatividad estadística de los coeficientes de regresión estandarizados fue analizada mediante un procedimiento bootstrap con 500 submuestras de 100 casos. La Tabla 3 resume los efectos directos e indirectos obtenidos y sus correspondientes niveles de significación. En la comprobación de los efectos indirectos (hipótesis 3, 5, 6, 7, 9 y 10), se aplicó la lógica de Zhao, Lynch y Chen (2010), quienes diferencian entre cinco patrones: (1) mediación complementaria, cuando existen efectos directos e indirectos en la misma dirección; (2) mediación competitiva, cuando existen efectos directos e indirectos en direcciones opuestas; (3) efectos indirectos únicos, cuando no existen efectos directos; (4) efectos directos únicos, cuando no existen efectos indirectos; y (5) inexistencia de efectos directos e indirectos.

Tabla 3. Ajuste del modelo estructural.

\begin{tabular}{lll}
\hline Coeficiente & $\begin{array}{l}\text { Efectos } \\
\text { directos }\end{array}$ & $\begin{array}{l}\text { Efectos } \\
\text { indirectos }\end{array}$ \\
\hline Intención $\rightarrow$ Conducta & $.43^{* * *}$ & -- \\
Intereses $\rightarrow$ Intención & $.26^{* * *}$ & -- \\
Intereses $\rightarrow$ Conducta & -- & $.12^{*}$ \\
Autoeficacia $\rightarrow$ Resultados extrínsecos & $.31^{* * *}$ & -- \\
Autoeficacia $\rightarrow$ Resultados intrínsecos & $.25^{* *}$ & -- \\
Autoeficacia $\rightarrow$ Intereses & $.39^{* * *}$ & $.09 *$ \\
Autoeficacia $\rightarrow$ Intención & $.35^{* * *}$ & .16 \\
Autoeficacia $\rightarrow$ Conducta & $.24^{* *}$ & .18 \\
Resultados extrínsecos $\rightarrow$ Intereses & .01 & -- \\
Resultados intrínsecos $\rightarrow$ Intereses & $.33^{* * *}$ & -- \\
Resultados extrínsecos $\rightarrow$ Intención & .06 & .01 \\
Resultados intrínsecos $\rightarrow$ Intención & .03 & $.09 *$ \\
Resultados extrínsecos $\rightarrow$ Conducta & .04 & .03 \\
Resultados intrínsecos $\rightarrow$ Conducta & .11 & .01 \\
\hline$* p<.05 ; * x<.01 ; * * \phi .001(t$ de Student con 499 grados de libertad).
\end{tabular}

De acuerdo al planteamiento de la hipótesis 1, se confirmó un efecto positivo directo de la intención sobre la conducta emprendedora temprana $(\beta=.43, p<.001)$. Asimismo, en confirmación de las hipótesis 2 y 3 , se dio un efecto positivo directo de los intereses sobre la intención $(\beta$ $=.26, p<.001)$ e indirecto sobre la conducta por mediación de la intención $(\beta=.12, p<.05)$.
En relación a la autoeficacia emprendedora, se comprobó un doble efecto positivo directo sobre las expectativas de resultados extrínsecos $(\beta=.31, p<.001)$ e intrínsecos $(\beta=$ $.25, p<.01)$, dando cumplimiento a la hipótesis 4 . Por su parte, la hipótesis 5 recibió respaldo empírico en su mayor parte, resultando significativos los efectos positivos directos $(\beta=.39, p<.001)$ e indirectos $(\beta=.09, p<.05)$ de la autoeficacia sobre los intereses emprendedores. No obstante, cuando los efectos indirectos vía expectativas de resultados extrínsecos e intrínsecos fueron considerados por separado, únicamente resultó significativa la mediación complementaria de éstos últimos. En la misma línea, las hipótesis 6 y 7 fueron parcialmente confirmadas, hallándose un efecto positivo directo de la autoeficacia sobre la intención $(\beta=.35, p$ $<.001)$ y conducta emprendedora temprana $(\beta=.24, p<$ .01 ), al tiempo que los efectos indirectos totales esperados en cada relación no resultaron significativos. No obstante, cuando se analizó por separado cada vía de efectos indirectos, se confirmó la mediación complementaria de los intereses en la relación entre autoeficacia e intención $(\beta=.10, p<$ $.001)$, y de los intereses e intenciones en la relación entre autoeficacia y conducta $(\beta=.16, p<.01)$.

Por lo que respecta a las hipótesis 8,9 y 10, relativas a los efectos de las expectativas de resultados en el modelo, se encontró un apoyo bastante limitado, dependiendo de la naturaleza de las recompensas esperadas a nivel extrínseco o intrínseco. Si bien no pudo confirmarse ningún tipo de efecto directo o indirecto de las expectativas de resultados extrínsecos sobre los intereses, intenciones y conductas emprendedoras tempranas, sí pudo evidenciarse un triple efecto de las expectativas de resultados intrínsecos en el modelo. En primer lugar, de acuerdo a la hipótesis 8, el efecto positivo directo sobre los intereses emprendedores resultó significativo $(\beta=.33, p<.001)$. En segundo lugar, se apreció un efecto únicamente indirecto sobre la intención de emprender, por mediación de los intereses $(\beta=.09, p<.05)$, lo que supone un cierto respaldo a la hipótesis 9 . Y tercero, si bien los efectos directos e indirectos totales de las expectativas de resultados intrínsecos sobre la conducta emprendedora temprana no resultaron significativos tal como se planteó en la hipótesis 10, cuando cada vía indirecta se consideró por separado, pudo apreciarse la existencia de una relación indirec- 
ta entre dichas variables por mediación de los intereses e intenciones $(\beta=.04, p<.05)$.

En el contexto de tales resultados, la Figura 2 resume los efectos directos e índices de ajuste global del modelo. A este último respecto, los índices $R^{2}$ superaron el mínimo recomendado de .10 (Falk y Miller, 1992) en cuatro de las cinco variables endógenas, de forma que el modelo global permitió explicar el 34\% de la varianza en los intereses emprendedores, el $33 \%$ en la intención de emprender, y el 31\% en la conducta emprendedora temprana. En el caso de las expectativas de resultados extrínsecos e intrínsecos, se obtuvieron unos índices $\mathrm{R}^{2}$ de .10 y .06 , respectivamente, revelando una limitada explicación de dichas variables en el conjunto del modelo.
Como complemento al estadístico $R^{2}$, algunos autores recomiendan el test Stone-Geisser (Geisser, 1974; Stone, 1974) en la estimación del ajuste global del modelo en términos de relevancia predictiva (Wold, 1982). Básicamente, dicho test realiza una estimación jacknife a través del estadístico $Q^{2}$, cuyos valores mínimos recomendados han de ser positivos y superiores a .20 (Chin 1998b). Ambos requisitos fueron satisfechos para los intereses emprendedores $\left(Q^{2}=\right.$ .20), la intención emprendedora $\left(Q^{2}=.24\right)$ y la conducta emprendedora temprana $\left(Q^{2}=.20\right)$. Por el contrario, y en coherencia con los resultados previamente descritos, el modelo arrojó una débil explicación de las expectativas de recompensa en el modelo predictivo.

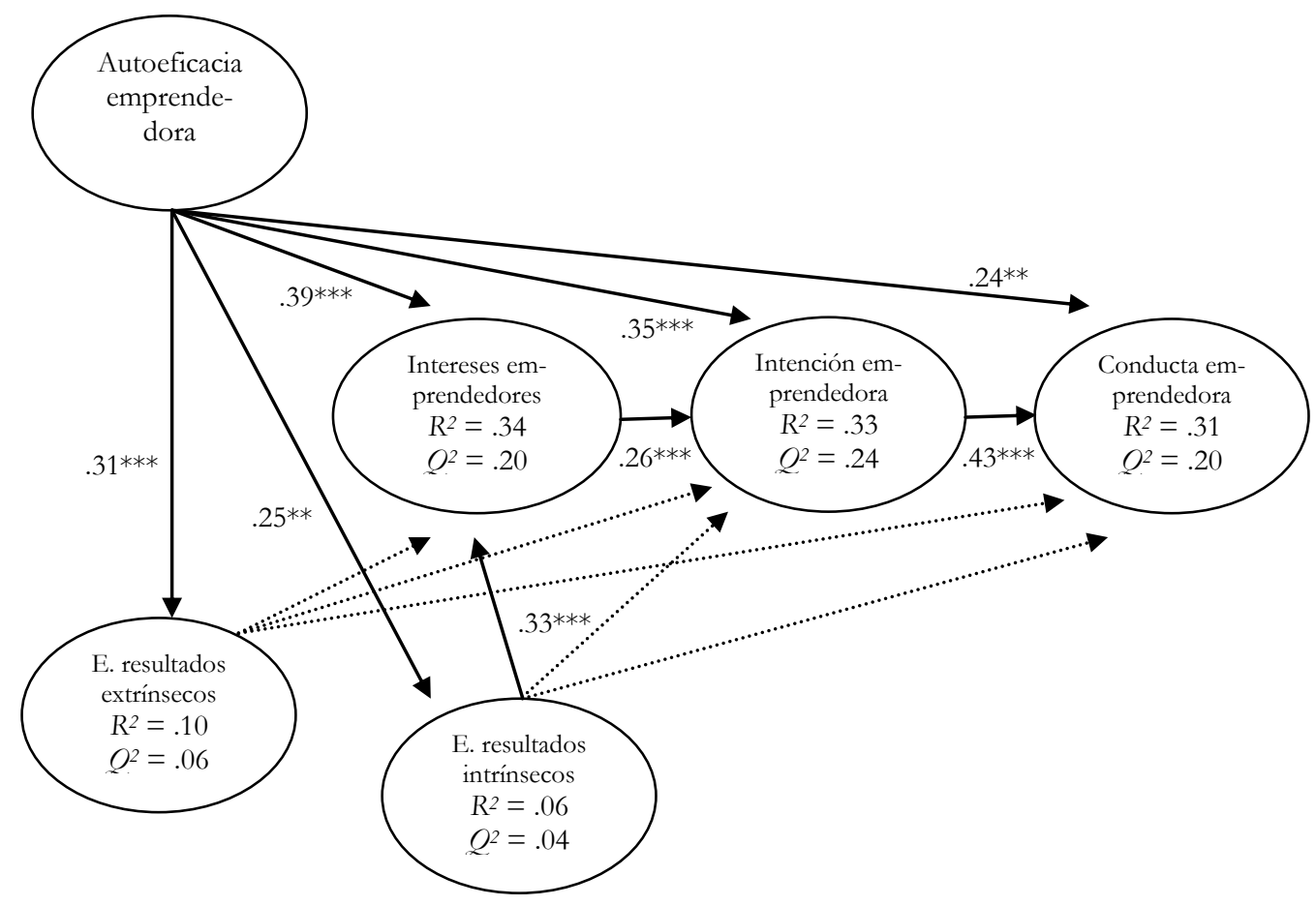

Figura 2. Modelo empírico. ${ }^{* *} p<.01 ; * * * p<.001$.

\section{Discusión y conclusiones}

En un intento de superar las limitaciones identificadas en la literatura previa, este trabajo ha propuesto un modelo explicativo de la iniciativa emprendedora del colectivo universitario a partir de los postulados de la TSCC de Lent et al. (1994, 2000). Desde este encuadre, y de forma coherente con la larga tradición de modelos teóricos de intencionalidad empresarial que erigen tal dimensión como principal antecedente de la conducta emprendedora planificada (Ajzen, 1991; Shapero, 1982), pudo constatarse un efecto positivo de la intención de emprender sobre el grado de implicación manifestado por el alumnado en actividades tempranas de puesta en marcha de una idea de negocio. Con todo, si bien la escasa prevalencia de empresas creadas por recién graduados superiores en los países de nuestro entorno y la complejidad técnica de llevar a cabo estudios longitudinales han dificultado a menudo la puesta a prueba de tal predicción en colectivos universitarios (Soutaris et al., 2007), el presente estudio supone una primera evidencia de que el compromiso experimentado por los individuos con respecto al autoempleo como opción laboral está estrechamente relacionado con la implementación de acciones específicas de iniciación en los negocios, en una fase previa a la constitución de la empresa como tal. Por tanto, mientras resulta poco probable que los estudiantes se embarquen en la creación de una empresa a la finalización de sus estudios, sí es esperable que aquéllos con más intención de emprender en el futuro co- 
miencen a mostrar cierto grado de implicación en conductas preparatorias, lo que plantea la conveniencia de prestar una mayor atención a la detección precoz de las inquietudes emprendedoras tempranas de los universitarios, para que puedan ser fácilmente reforzadas como potenciales iniciativas empresariales a largo plazo. De hecho, esta perspectiva práctica está siendo asumida por programas pioneros de creación de empresas instaurados en la última década en algunas universidades españolas, desde los que se contemplan actuaciones como talleres de generación y maduración de ideas o cursos de introducción a la gestión empresarial dirigidos a acercar a la fase de pre-empresarización a unos destinatarios en los que se presupone cierta motivación emprendedora inicial (Placer y Vázquez, 2005).

En el mismo orden de cosas, una de las aportaciones más novedosas de la aproximación asumida en este trabajo con respecto a la literatura previa sobre comportamiento emprendedor en la juventud remite a la consideración de los intereses vocacionales como antecedentes del proceso de elección de carrera, en una visión más cercana a la realidad profesional característica del alumnado universitario. Desde este punto de vista, el trabajo aquí discutido extiende la investigación previa sobre el desarrollo de vocaciones emprendedoras en la adolescencia (Bonnett y Furnham, 1991; Schmitt-Rodermund, 2004; Schmitt-Rodermund y Vondracek, 2002, Schröder y Schmitt-Rodermund, 2006) a etapas posteriores y más próximas al periodo de transición al mundo laboral, encontrando un efecto positivo directo de dicha variable sobre la intención de emprender e indirecto sobre la conducta por mediación de la intención. Con todo, estos resultados apuntan a que los patrones de atracción temprana de los estudiantes hacia la actividad emprendedora desempeñan un papel de gran importancia en la conducción de sus propósitos profesionales hacia la creación de empresas, ofreciendo una visión más completa de la progresión de metas de carrera emprendedora en la etapa universitaria. Desde esta perspectiva, si bien los modelos previos (Ajzen, 1991; Shapero, 1982) se han centrado en el estudio de cuestiones de deseabilidad y viabilidad conductual en la explicación de la intencionalidad emprendedora en la juventud, este trabajo apunta a la conveniencia de estudiar dichos determinantes en interacción con otros procesos tempranos de elección vocacional que aparecen asociados a intenciones y conductas posteriores.

Por lo que respecta a los desencadenantes de las metas emprendedoras, el modelo planteado asumió que los intereses, intenciones y conductas emprendedoras tempranas estarían asociados a la autoeficacia y expectativas de resultados anticipadas en la creación de empresas, estando a su vez estas variables positivamente relacionadas entre sí. A tal respecto, en la línea de lo sugerido por la literatura previa (Boyd y Vozikis, 1994; Chen et al., 1998; De Noble et al., 1999), la autoeficacia tuvo un efecto positivo directo sobre las expectativas de resultados extrínsecos e intrínsecos, en apoyo a la conclusión general de que las percepciones de competencia emprendedora se asocian con las anticipaciones instrumenta- les de alcanzar resultados deseados mediante la creación de una empresa. Asimismo, los resultados apoyaron un modelo de efectos directos de la autoeficacia sobre el desarrollo de metas emprendedoras, pudiendo constatarse sólo parcialmente los efectos indirectos sobre los intereses -por mediación de las expectativas de resultados intrínsecos-, la intención -por mediación de los intereses-, y la conducta emprendedora temprana -por mediación de los intereses y la intención-. En la línea de trabajos previos, (De Noble et al., 1999; Kickul et al., 2009; Moriano, Topa et al., 2012; Wilson et al., 2007; Zhao et al., 2005; Zellweger et al., 2011), este hallazgo apunta a la conclusión de que el sentido de competencia asociado a la percepción de viabilidad de la conducta emprendedora constituye entre los futuros titulados el principal predictor de su implicación en tales propósitos. Desde esta perspectiva, y siguiendo las prescripciones de Bandura (1986, 1997), cabe retomar aquí la utilidad de basar la educación de la iniciativa emprendedora en contextos universitarios en prácticas pedagógicas facilitadoras de las cuatro fuentes fundamentales de autoeficacia percibida basadas en la ejecución personal exitosa, el aprendizaje vicario o modelado, la persuasión social, y la inferencia de reacciones fisiológicas (Wilson et al., 2007; Zhao et al., 2005).

Por su parte, y a la vista de los resultados obtenidos en este trabajo, la relación entre expectativas de resultados y desarrollo de proyecciones emprendedoras parece ser bastante limitada y depende en gran medida de la naturaleza de las recompensas anticipadas en tal ocupación profesional (Mitchell y Knudsen, 1993). De este modo, mientras no pudo constatarse ningún tipo de asociación entre expectativas de resultados extrínsecos y metas emprendedoras, sí resulto significativo el efecto directo de las expectativas de resultados intrínsecos sobre los intereses emprendedores, así como indirecto sobre la intención -por mediación de los interesesy, en menor medida, sobre la conducta de iniciación empresarial -por mediación de los intereses y la intención-. Este patrón sugiere que el desarrollo de vocaciones emprendedoras tempranas está asociado a la consideración de la creación de empresas como alternativa profesional útil para conseguir recompensas personales inherentes a la propia actividad (i.e., satisfacción, autoestima, aprendizaje, etc.), dejándose ver sólo de forma tenue los efectos de esta asociación en la evolución hacia el proceso de elección de carrera. De esta forma, las expectativas de resultados intrínsecos no parecen ser factores decisivos en la configuración de intenciones y conductas tempranas de iniciación empresarial, de la misma forma que la anticipación de otro tipo de incentivos externos (e.g., retribución económica, autonomía, etc.) no representa una condición suficiente para canalizar a los estudiantes hacia vocaciones emprendedoras y propósitos acordes de carrera.

Con todo, puede decirse que existe una cierta desconexión entre las expectativas de resultados y el resto de variables del modelo, que se hace especialmente patente en las mayores puntuaciones medias obtenidas por los encuestados en las escalas de medición del constructo a nivel extrínseco e intrínseco, en comparación con el resto de medidas. La exis- 
tencia de este desajuste entre las actitudes positivas de los jóvenes con estudios superiores hacia la deseabilidad del autoempleo y su escasa tendencia a implicarse en metas de carrera al respecto en los países de nuestro entorno ha sido frecuentemente documentado en estudios previos (Dirección General de Empresa e Industria, 2012b; Ronald et al., 2013), al tiempo que diversas investigaciones dedicadas al análisis de los factores desencadenantes de la intencionalidad emprendedora avalan la idea de que los constructos asociados a la viabilidad conductual explican una mayor proporción de la varianza en el comportamiento emprendedor que las actitudes hacia los resultados empresariales (e.g., Fitzsimmons y Douglas, 2011; Kolvereid, 1996a; Krueger, 1993; Tkachev y Kolvereid, 1999; Townsend et al., 2010).

En la interpretación de tales hallazgos, el marco de la TSCC recoge los postulados de Bandura $(1986,1997)$ referidos al poder relativo de las expectativas de recompensa en la explicación del comportamiento humano frente a la autoeficacia percibida. Así, Lent et al. (1994) argumentan que en situaciones en las que la calidad del rendimiento de carrera garantiza la consecución de determinadas recompensas, la autoeficacia representa el principal factor determinante de la conducta vocacional, influyendo también sobre la representación de las propias consecuencias esperadas. Aplicado a los resultados del presente estudio, podría inferirse que, dada la fortaleza de las recompensas anticipadas por los futuros graduados en la creación de empresas, el grado de implicación en metas al respecto depende en última instancia de las percepciones de competencia personal para alcanzar tales resultados mediante la conducta emprendedora. Con todo, la tendencia de los resultados descritos en los párrafos precedentes apunta a la conclusión general de que el escaso desarrollo de propensiones hacia el autoempleo en el colectivo universitario responde fundamentalmente a la baja viabilidad asociada a dicha conducta en base a precepciones de eficacia personal. En otras palabras, el discurso típico del alumnado parece ser un "quiero, pero no puedo" en el que las actitudes positivas hacia los resultados emprendedores no desembocan adecuadamente en metas de carrera acordes a causa de los obstáculos percibidos en el proceso.

Más allá de las consideraciones previas, el alcance de los resultados obtenidos en el presente estudio debe ser interpretado ante el matiz de ciertas limitaciones que dejan la puerta abierta a futuras investigaciones en el campo. En primer lugar, cabe apuntar la propia novedad del marco teórico empleado en la aplicación de la TSCC al estudio de la conducta emprendedora estudiantil, así como el escaso desa- rrollo teórico de algunas de las variables centrales del modelo (i.e., los intereses emprendedores), siendo precisadas nuevas investigaciones que repliquen los resultados obtenidos o profundicen en el refinamiento de los constructos propuestos y su medición.

En segundo lugar, pese a la utilidad del estudio descrito en la comprensión de las inquietudes y proyecciones emprendedoras de los futuros graduados universitarios, la naturaleza transversal del diseño empleado condiciona que las conclusiones avanzadas deban interpretarse como evidencias de relaciones estadísticamente significativas entre variables en el contexto de los efectos identificados, más que como inferencias causales entre las mismas. A tal respecto, nuevos análisis longitudinales son requeridos para dar cuenta de la evolución de los procesos iniciales de elección de carreras emprendedoras en la forma constatable de nuevas empresas exitosas en el mercado. Unido a ello, y tomando como referencia los frecuentes obstáculos experimentados por los jóvenes para hacer realidad sus propósitos empresariales, cabe considerar la conveniencia de analizar el funcionamiento predictivo de la TSCC en relación a otro tipo de variables contextuales y barreras percibidas por los individuos, así como el papel de la propia agencia personal en la neutralización de tales influencias (Lent et al., 2001).

Por último, pese a que no ha de pasar desapercibido el esfuerzo invertido en la selección de los participantes en la investigación a partir de un procedimiento de muestreo estratificado basado en la distribución real del alumnado por ramas de enseñanza, cabe apuntar que la complejidad entrañada por el procedimiento de recogida de información derivó finalmente en el recurso a muestras de conveniencia, a partir de la disponibilidad práctica de profesores y alumnos con suficiente experiencia universitaria dispuestos a colaborar voluntariamente en la investigación. Con todo, futuros intentos deberían estar destinados a procurar una mayor representatividad muestral en base al cruce de información académica y sociodemográfica. En la misma línea, nuevos trabajos son requeridos en la generalización de los resultados a otras instituciones nacionales y europeas, dando cabida incluso a otros modelos de educación superior con el propósito de ganar una mayor precisión en la identificación de los factores condicionantes de la iniciativa emprendedora en la juventud universitaria.

Notas.-La investigación descrita en este trabajo ha sido realizada con el respaldo del Programa de Formación del Profesorado Universitario (FPU) del Ministerio de Educación.

\section{Referencias}

Ajzen, I. (1987). Attitudes, traits, and actions: Dispositional prediction of behavior in social psychology. Advances in Experimental Social Psychology, 20, 1-63.

Ajzen, I. (1991). The theory of planned behavior. Organizational Behavior and Human Decision Processes, 50, 179-211.

Ajzen, I. (2001). Nature and operation of attitudes. Annual Review of Psychology, 52, 27-58.
Ajzen, I. (2002). Perceived behavioral control, self-efficacy, locus of control, and the theory of planned behavior. Journal of Applied Social Psychology, 32, 665683.

Ajzen, I. y Fishbein, M. (1980). Understanding Attitudes and Predicting Social Bebavior. Englewood Cliffs, NJ: Prentice Hall. 
Aldrich, H. E. y Martínez, M. A. (2001). Many are called but few are chosen: An evolutionary perspective for the study of entrepreneurship. Entrepreneurship Theory and Practice, 25(4), 41-56.

Alemany, L., Álvarez, C., Planellas, M. y Urbano, D. (2011). Libro Blanco de la Iniciativa Emprendedora en España. ESADE Entrepreneurship Institute.

Alsos, G. A. y Kolvereid, L. (1998). The business gestation process of novice, serial and parallel business founders. Entrepreneurship Theory and Practice, 22(4), 101-114.

Arenius, P. y Minniti, M. (2005). Perceptual variables and nascent entrepreneurship. Small Business Economics, 24, 233-247.

Autio, E., Keeley, R. H., Klofsten, M., Parker, G. G. C. y Hay, M. (2001). Entrepreneurial intent among students in Scandinavia and in the USA. Enterprise and Innovation Management Studies, 2, 145-160.

Bagozzi, R. P. y Yi, Y. (1991). Multitrait-multimethod matrices in consumer research. Journal of Consumer Research, 17, 426-439.

Bandura, A. (1977). Self-efficacy: Toward a unifying theory of behavioural change. Psychological Review, 84, 191-215.

Bandura, A. (1986). Social Foundations of Thought and Action: A Social Cognitive Theory. Englewood Cliffs, NJ: Prentice-Hall.

Bandura, A. (1997). Self-efficacy: The Exercise of Control. Nueva York: Freeman.

Barclay, D., Higgins, C. y Thompson, R. (1995). The Partial Least Squares (PLS) approach to causal modelling: Personal computer adoption and use as an illustration. Technology Studies, 2, 285-309.

Betz, N. E. (2008). Advances in vocational theories. En S. D. Brown y R. W. Lent (Eds.), Handbook of Counseling Psychology (4 ed., pp. 357-374). Nueva York: Wiley.

Bird, B. (1988). Implementing entrepreneurial ideas: The case for intentions. Academy of Management Review, 13, 442-454.

Blanch Ribas, J. M. (2005). Estudio de calidad de vida laboral en el subempleo. Psicología de las Organizaciones, del Trabajo y Recursos Humanos y de la Salud, 4, 7988.

Blanco, A. (2009). El modelo cognitivo social del desarrollo de la carrera: Revisión de más de una década de investigación empírica. Revista de Educación, $350,423-445$.

Bollen, K. A. (1989). Structural Equations with Latent Variables. Nueva York: Wiley.

Bonnett, C. y Furnham, A. (1991). Who wants to be an entrepreneur? A study of adolescents interested in a Young Enterprise scheme. Journal of Economic Psychology, 12, 465-478.

Boyd, N. y Vozikis, G. S. (1994). The influence of self-efficacy on the development of entrepreneurial intentions and actions. Entrepreneurship Theory and Practice, 14(1), 63-77.

Brenner, O. C., Pringle, C. D. y Greenhaus, J. H. (1991). Perceived fulfillment of organizational employment versus entrepreneurship: Work values and career intentions of business college graduates. Journal of Small Business Management, 29(3), 62-74.

Carter, N. M., Gartner, W. B. y Reynolds, P. D. (1996). Exploring start-up event sequences. Journal of Business Venturing, 11, 151-166.

Carter, N. M., Gartner, W. B., Shaver, K. G. y Gatewood, E. J. (2003). The career reasons of nascent entrepreneurs. Journal of Business Venturing, 18, 13-39.

Cassar, G. (2007). Money, money, money? A longitudinal investigation of entrepreneur career reasons, growth preferences and achieved growth. Entrepreneurship and Regional Development, 19, 89-107.

Chandler, G. N. y Lyon, D. W. (2001). Issues of research design and construct measurement in entrepreneurship research: The past decade. Entrepreneurship Theory and Practice, 25(4), 101-113.

Chen, C. C., Greene, P. G. y Crick, A. (1998). Does entrepreneurial self-efficacy distinguish entrepreneurs from managers? Journal of Business Venturing, 13, 295-316.

Chin, W. W. (1998a). Issues and opinion on structural equation modeling. MIS Quarterly, 22(1), 7-16.

Chin, W. W. (1998b). The partial least squares approach to structural equation modeling. En A. George (Ed.), Modern Methods for Business Research (pp. 295336). New Jersey: Laurence Erlbaum Associates.

Chin, W. W., Marcolin, B. L. y Newsted, P. R. (2003). A partial least squares latent variable modeling approach for measuring interaction effects: Results from a Monte Carlo simulation study and an electronic mail emotion/adoption study. Information Systems Research, 14, 189-217.

Collins, L., Hannon, P. D. y Smith, A. (2004). Enacting entrepreneurial intent: The gaps between student needs and higher education capability. Education + Training, 46, 454-463.

Comisión Europea (2006). Entrepreneurship Education in Europe: Fostering Entrepreneurial Mindsets through Education and Learning. Final proceedings. Oslo: Autor.

Corduras, A., Urbano, D., Rojas, A. y Martínez, S. (2008). The relationship between University support to entrepreneurship with entrepreneurial activity in Spain. International Advances in Economic Research, 16, 395-406.
Davidsson, P. y Honig, B. (2003). The role of social and human capital among nascent entrepreneurs. Journal of Business Venturing, 18, 301-331.

De Noble, A., Jung, D. y Ehrlich, S. (1999). Entrepreneurial self-efficacy: The development of a measure and its relationship to entrepreneurial action. En R. D. Reynolds, W. D. Bygrave, S. Manigart, C. M. Mason, G. D. Meyer, H. J. Sapienza y K. G. Shaver (Eds.), Frontiers of Entrepreneurship Research (pp. 7378). Waltham, MA: P \& R Publication Inc.

Díaz-Casero, J. C., Ferreira, J. J., Hernández-Mogollón, R. y Raposo, M. (2012). Influence of institutional environment on entrepreneurial intention: A comparative study of two countries university students. International Entrepreneurship and Management Journal, 8(1), 55-74.

Diegelman, N. M. y Subich, L. M. (2001). Academic and vocational interests as a function of outcome expectancies in Social Cognitive Career Theory. Journal of Vocational Behavior, 59, 394-405.

Dirección General de Empresa e Industria (2008). La Iniciativa Emprendedora en la Enseñanza Superior, especialmente en Estudios No Empresariales. Bruselas: Comisión Europea.

Dirección General de Empresa e Industria (2012a). Effects and Impact of Entrepreneurship Programmes in Higher Education. Bruselas: Comisión Europea.

Dirección General de Empresa e Industria (2012b). Entrepreneurship in the EU and Beyond. Bruselas: Comisión Europea.

Douglas, E. J. y Sheperd, D. A. (2002). Self-employment as a career choice: Attitudes, entrepreneurial intentions, and utility maximization. Entrepreneurship Theory and Practice, 26(3), 81-90.

Edelman, L. F., Brush, C. B., Manolova, T. S. y Greene, P. G. (2010). Start-up motivations and growth intentions of minority nascent entrepreneurs. Journal of Small Business Management, 48, 174-196.

Eurostat (2011). Labour Market Statistics. Luxemburgo: Oficina de Publicaciones de la Unión Europea.

Falk, R. F. y Miller, N. B. (1992). A Primer for Soft Modeling. Akron, Ohio: The University of Akron.

Fayolle, A. (2005). Evaluation of entrepreneurship education: Behavior performing or intention increasing? International Journal Entrepreneurship and Small Business, 2, 89-98.

Ferry, R. R., Fouad, N. A. y Smith, P. L. (2000). The role of family context in a social cognitive model for career-related choice behavior: A Math and Science perspective. Journal of Vocational Behavior, 57, 348-364.

Fiet, J. O. y Pankaj, P. (2008). Prescriptive Entrepreneurship. Cheltenham, UK: Edward Elgar.

Fiske, D. W. (1982). Convergent-discriminant validation in measurements and research strategies. En D. Brinbirg y L. H. Kidder (Eds.), Forms of V alidity in Research (pp. 77-92). San Francisco: Jossey-Bass.

Fitzsimmons, J. R. y Douglas, E. J. (2011). Interaction between feasibility and desirability in the formation of entrepreneurial intentions. Journal of Business Venturing, 26(4), 431-440.

Flores, L. Y., Robitschek, C., Celebi, E., Andersen, C. y Hoang, U. (2010). Social cognitive influences on Mexican Americans' career choices across Holland's themes. Journal of Vocational Behavior, 76, 198-210.

Fornell, C. y Bookstein, F. L. (1982). A comparative analysis of two structural equation models: Lisrel and PLS applied to market data. En C. Fornell (Ed.), A Second Generation of Multivariate Analysis (Vol. 1, pp. 289-324). Nueva York: Praeger Publishers.

Fornell, C. y Larcker, D. F. (1981). Evaluating structural equation models with unobservable variables and measurement error. Journal of Marketing Research, 18, 39-50.

Gainor, K. A. y Lent, R. W. (1998). Social cognitive expectations and racial identity attitudes in predicting the Math choice intentions of black college students. Journal of Counseling Psychology, 45, 403-413.

Galloway, L., Anderson, M., Brown, W. y Wilson, L. (2005). Enterprise skills for the economy. Education + Training, 47, 7-17.

García-Montalvo, J. y Peiró, J. M. (2009). Análisis de la Sobrecualificación y la Flexibilidad Laboral. Observatorio de Inserción Laboral de los Jóvenes 2008. Valencia: Fundación Bancaja-IVIE.

García-Montalvo, J. y Peiró, J. M. (2011). Crisis Económica e Inserción Laboral de los Jóvenes. Resultados del Observatorio de Inserción Laboral de los Jóvenes 2011. Valencia: Fundación Bancaja-IVIE.

Gatewood, E. J., Shaver, K. G. y Gartner, W. B. (1995). A longitudinal study of cognitive factors influencing start-up behaviors and success at venture creation. Journal of Business Venturing, 10, 371-391.

Geisser, S. (1974). A predictive approach to the random effects model. Biometrica, 61, 101-107.

Gibb, A. A. (1983). The small business challenge to management education: Introduction. Distinguishing characteristics of small firms. Journal of European Industrial Training, 7(5), 3-41. 
Goethner, M., Obschonka, M., Silvereisen, R. K., Cantner, U. (2012). Scientists' transition to academic entrepreneurship: Economic and psychological determinants. Journal of Economic Psychology, 33(3), 628-641.

Guerrero, M., Rialp, J. y Urbano, D. (2008). The impact of desirability and feasibility on entrepreneurial intentions: A structural equation model. International Entrepreneurship and Management Journal, 4, 35-50.

Hair, J. F. J., Anderson, R. E., Tatham, R. L. y Black, W. C. (1998). Multivariate Data Analysis ( $5^{\mathrm{a}}$ ed.). Londres: Prentice Hall International.

Hansen, J. C. (1984). The measurement of vocational interests: Issues and future directions. En S. D. Brown y R. W. Lent (Eds.), Handbook of Counseling Psychology (pp. 99-136). Nueva York: Wiley.

Hechavarría, D. M., Renko, M. y Matthews, C. H. (2012). The nascent entrepreneurship hub: Goals, entrepreneurial self-efficacy and start-up outcomes. Small Business Economics 39, 685-701.

Hemmasi, M. y Hoelscher, M. (2005). Entrepreneurship research: Using students as proxies for actual entrepreneurs. Journal of Entrepreneurship Education, 8, 4959.

Hernández, R. y Corduras, A. (2013). Global Entrepreneurship Monitor. Informe GEM España 2012. Madrid: Red Española de Equipos Regionales GEM.

Hinkin, T. R. (1995). A review of scale development practices in the study of organizations. Journal of Management, 21, 967-988.

Holland, J. L. (1959). A theory of vocational choice. Journal of Counseling Psychology, 6, 35-45.

Holland, J. L. (1985). Making vocational choices: A theory of Vocational Personalities and Work Environments ( $2^{\mathrm{a}}$ ed.). Englewood Cliffs, NJ: Prentice-Hall.

Holland, J. L. (1997). Making Vocational Choices (3a ed.). Odessa, FL: Psychological Assessment Resources.

Hynes, B. (1996). Entrepreneurship education and training. Introducing entrepreneurship into non-business disciplines. Journal of European Industrial Training, 20(8), 10-17.

Johnson, D., Craig, J. B. L. y Hildebrand, R. (2006). Entrepreneurship education: Towards a discipline-based framework. Journal of Management Development, 25(1), 40-54.

Katz, J. A. (1992). A psychosocial cognitive model of employment status choice. Entrepreneurship Theory and Practice, 17(1), 29-37.

Katz, J. A. y Gartner, W. B. (1988). Properties of emerging organizations. Academy of Management Review, 13, 429-441.

Kickul, J., Gundry, L. K., Barbosa, S. D. y Whitcanack, L. (2009). Intuition versus analysis? Testing differential models of cognitive style on entrepreneurial self-efficacy and the new venture creation process. Entrepreneurship Theory and Practice 33, 439-453.

Kim, M. y Hunter, J. (1993). Relationships among attitudes, intentions and behavior. Communication Research, 20, 331-364.

Koellinger, P., Minniti, M. y Schade, C. (2007). "I think I can, I think I can": Overconfidence and entrepreneurial behavior. Journal of Economic Psychology, $28,502-528$.

Kolvereid, L. (1996a). Prediction of employment status choice intentions. Entrepreneurship Theory and Practice, 20(3), 47-57.

Kolvereid, L. (1996b). Organizational employment versus self-employment: Reasons for career choice intentions. Entrepreneurship Theory and Practice, 20(3), 23-32.

Kolvereid, L., \& Isaksen, E. (2006). New business start-up and subsequent entry into self-employment. Journal of Business Venturing, 21, 866-885.

Krueger, N. F. (1993). The impact of prior entrepreneurial exposure on perceptions of new venture feasibility and desirability. Entrepreneurship Theory and Practice, 18(1), 5-21.

Krueger, N. F. (2000). The cognitive infrastructure of opportunity emergence. Entrepreneurship Theory and Practice, 24(3), 5-23.

Krueger, N. F. y Brazeal, D. V. (1994). Entrepreneurial potential and potential entrepreneurs. Entrepreneurship Theory and Practice, 18(3), 91-104.

Krueger, N. F. y Carsrud, A. L. (1993). Entrepreneurial intentions: Applying the theory of planned behavior. Entrepreneurship and Regional Development, 5, 31530 .

Krueger, N. F., Reilly, M. D. y Carsrud, A. L. (2000). Competing models of entrepreneurial intentions. Journal of Business Venturing, 15, 411-432.

Lanero, A., Vázquez, J. L., Gutiérrez, P. y García, M. P. (2011). The impact of entrepreneurship education in European universities: An intention-based approach analyzed in the Spanish area. International Review on Public and Nonprofit Marketing, 8(2), 111-130.

Lee, S. H. y Wong, P. K. (2004). An exploratory study of technopreneurial intentions: A career anchor perspective. Journal of Business Venturing, 19, 7-28.

Lent, R. W. y Brown, S. D. (2006). Integrating person and situation perspectives on work satisfaction: A social-cognitive view. Journal of Vocational Behavior, 69, 236-247.
Lent, R. W., Brown, S. D., Brenner, B. R., Chopra, S. B., Davis, T., Talleyrand, R. y Suthakaran, V. (2001). The role of contextual supports and barriers in the choice of Math/Science educational options: A test of social cognitive hypotheses. Journal of Counseling Psychology, 48, 474-483.

Lent, R. W., Brown, S. D. y Hackett, G. (1994). Toward a unifying social cognitive theory of career and academic interest, choice, and performance. Journal of Vocational Behavior, 45, 79-122.

Lent, R. W., Brown, S. D. y Hackett, G. (2000). Contextual supports and barriers to career choice: A social cognitive analysis. Journal of Counseling Psychology, 47, 36-49.

Lent, R. W., Brown, S. D., Schmidt, J. S., Brenner, B. R., Lynos, H. y Treistman, D. (2003). Relation of contextual supports and barriers to choice behaviour in engineering majors. Test of alternative social cognitive models. Journal of Counseling Psychology, 50, 458-465.

Lent, R. W., Brown, S. D., Sheu, H. B., Schmidt, J. S., Brenner, B. R., Gloster, C. S., Wilkins, G., Schmidt, L. C., Lynos, H. y Treistman, D. (2005). Social cognitive predictors of academic interests and goals in engineering: Utility for women and students at historically black universities. Journal of Counseling Psychology, 52, 84-92.

Lent, R. W., López, A. M., López, F. G. y Sheu, H. B. (2008). Social Cognitive Career Theory and the prediction of interests and choice goals in the computing disciplines. Journal of Vocational Behavior, 73, 52-62.

Lent, R. W., López, F. G., Sheu, H. B. y López, A. M. (2011). Social cognitive predictors of the interests and choices of computing majors: Applicability to underrepresented students. Journal of Vocational Behavior, 78(2), 184-192.

Lent, R. W., Sheu, H. B., Gloster, C. S. y Wilkins, G. (2010). Longitudinal test of the social cognitive model of choice in engineering students at historically Black universities. Journal of Vocational Behavior, 76, 355-365.

Lent, R. W., Sheu, H. B., Singley, D., Schmidt, J. A., Schmidt, L. C. y Gloster, C. S. (2008). Longitudinal relations of self-efficacy to outcome expectations, interests, and major choice goals in engineering students. Journal of Vocational Behavior, 73, 328-335.

Levenburg, N. M., Lane, P. M., Schwarz, T. V. y Rapids, G. (2006). Interdisciplinary dimensions in entrepreneurship. Journal of Education for Business, 81, 275 282.

Liao, H. Y., Armstrong, P. I. y Rounds, J. (2008). Development and initial validation of public domain Basic Interest Markers. Journal of Vocational Behavior, 73, 159-183.

Liñán, F. y Chen, Y. (2009). Development and cross-cultural application of a specific instrument to measure entrepreneurial intentions. Entrepreneurship Theory and Practice, 33, 593-617.

Liñán, F., Urbano, D. y Guerrero, M. (2011). Regional variations in entrepreneurial cognitions: Start-up intentions of university students in Spain. Entrepreneurship and Regional Development, 23(3-4), 187-215.

MacMillan, I. y Katz, J. A. (1992). Idiosyncratic milieus of entrepreneurship research: The need for comprehensive theories. Journal of Business Venturing, 7, 1-8.

Markman, G. D., Balkin, D. B. y Baron, R. A. (2002). Inventors and new venture formation: The effects of general self-efficacy and regretful thinking. Entrepreneurship Theory and Practice, 27, 149-165.

Markman, G. D., Baron, R. A. y Balkin, D. B. (2005). Are perseverance and selfefficacy costless? Assessing entrepreneurs' regretful thinking. Journal of Organizational Behavior, 26, 1-19.

Martínez, R. (1995). Psicometría: Teoría de los Test Psicológicos y Educativos. Madrid: Síntesis.

McGee, J. E., Mueller, S. L. y Sequeira, J. M. (2009). Self-efficacy: Refining the measure. Entrepreneurship Theory and Practice, 33, 965-988.

McLarty, R. (2005). Entrepreneurship among graduates: Towards a measured response. Journal of Management, 24, 223-238.

McMullan W. E. y Long, W. A. (1987). Education in the nineties. Journal of Business Venturing. 2, 261-275.

Mitchell, T. R. y Knudsen, B. W. (1973). Instrumentality theory predictions of students' attitudes towards business and their choice of business as an occupation. The Academy of Management Journal, 16, 41-52.

Moriano, J. A, Gorgievski, M., Laguna, M., Stephan, U. y Zarafshani, K. (2012). Cross-cultural approach to understanding entrepreneurial intention. Journal of Career Development, 39(2), 162-185.

Moriano, J. A., Palací, F. J. y Morales, J. F. (2006). Adaptation and validation of the entrepreneurial self-efficacy scale in Spain. Revista de Psicologia Social, 21, 51-64.

Moriano, J. A., Topa, G., Molero, F., Entenza, A. M. y Lévy-Mangin, J.-P. (2012). Autoeficacia para el liderazgo emprendedor. Adaptación y validación de la escala CESE en España. Anales de Psicología, 28(1), 171-179.

Muñoz Adánez, A. (1997). El perfil del emprendedor y el perfil del universitario español en relación con el mismo. En A. Muñoz Adánez y F. Cachón (Eds.), 
Proyecto Emprendizaje: Cuatro estudios sobre la Universidad española. Madrid: Euroforum-Fundación General de la UCM.

Muñoz Adánez, A. (1999). ¿Es emprendedor el universitario español? En A. Muñoz Adánez y M. Bautista (Eds.), El Espiritu Emprendedor y la Creación de Empresas (pp. 1-14). Madrid: Editorial Complutense.

Nunnally, J. C. (1978). Psychometric Theory. Nueva York: McGraw-Hill.

Nunnally, J. C. y Bernstein, I. H. (1994). Psychometric theory (3 ${ }^{a}$ ed.). Nueva York; McGraw-Hill.

Organización para la Cooperación y el Desarrollo Económicos. (2011). Education at a Glance. OECD indicators. París: OCDE.

Peiró, J. M., Sora, B. y Caballer, A. (2012). Job insecurity in the younger Spanish workforce: Causes and consequences. Journal of Vocational Behavior, 80(2), 444-453.

Placer, J. L. y Vázquez, J. L. (2005). Actividades y resultados del Plan Legio en 2002-05 y proyección para 2006. León: Secretariado de Publicaciones y Secretariado de Promoción Empresarial de la Universidad de León.

Podsakoff, P. M., MacKenzie, S. B., Lee, J. y Podsakoff, N. P. (2003). Common method biases in behavioral research: A critical review of the literature and recommended remedies. The Journal of Applied Psychology, 88, 879-903.

Renko, M., Kroeck, K. G., y Bullough, A. (2012). Expectancy theory and nascent entrepreneurship. Small Business Economics, 39(3), 667-648.

Reynolds, P. D. y Miller, P. B. (1992). New firm gestation: Conception, birth, and implications for research. Journal of Business Venturing, 7, 405-417.

Ringle, C. M., Wende, S. y Will, A. (2005). SmartPLS 2.0 (beta). Hamburg: University of Hamburg.

Robinson, P. B., Huefner, J. C. y Hunt, H. K. (1991). Entrepreneurial research on student subjects does not generalize to real world entrepreneurs. Journal of Small Business Management, 29(2), 42-50.

Ronald, S., Kelley, D., Kew, J., Herrington, M. y Vorderwülbecke, A. (2013). Global Entrepreneurship Monitor. 2012 Executive Report. Babson Park: Babson College.

Rotefos, B. y Kolvereid, L. (2005). Aspiring, nascent and fledgling entrepreneurs: An investigation of the business start-up process. Entrepreneurship and Regional Development, 17, 109-127.

Ruíz, J., Solé, F. y Veciana, J. M. (2004). Creación de empresas y universidad (1ª ed.). Cádiz: Fundación Universidad-Empresa de la provincia de Cádiz.

Schaub, M. y Tokar, D. M. (2005). The role of personality and learning experiences in Social Cognitive Career Theory. Journal of Vocational Behavior, 66, 304-325.

Schmitt-Rodermund, E. (2004). Pathways to successful entrepreneurship: Parenting, personality, early entrepreneurial competence, and interests. Journal of Vocational Behavior, 65, 498-518.

Schmitt-Rodermund, E. y Vondracek, F. W. (2002). Occupational dreams, choices and aspirations: Adolescents' entrepreneurial prospects and orientations. Journal of Adolescence, 25, 65-78.

Schröder, E. y Schmitt-Rodermund, E. (2006). Crystallizing enterprising interests among adolescents through a career development program: The role of personality and family background. Journal of Vocational Behavior, 69, 494-509.

Schwab, D. P. (1980). Construct validity in organization capital perspective. En B. M. Staw y L. L. Cummings (Eds.), Research In Organizational Behavior (Vol. 2, pp. 3-43). Greenwich, CT: JAI Press.

Sequeira, J. M., Mueller, S. L. y McGee, J. E. (2007). The influence of social ties and self-efficacy in forming entrepreneurial intentions and motivating nascent behavior. Journal of Development Entrepreneurship, 12, 275-293.
Sexton, D. L. y Bowman, N. B. (1984). Entrepreneurship education suggestions for increasing effectiveness. Journal of Small Business Management, 22(2), 18-25.

Shane, S. y Venkataraman, S. (2000). The promise of entrepreneurship as a field of research. Academy of Management Review, 25, 217-226.

Shapero, A. (1982). The social dimensions of entrepreneurship. En C. Kent, D. Sexton y K. Vesper (Eds), The Encyclopedia of Entrepreneurship (pp. 72-90). Englewood Cliffs, NJ: Prentice-Hall.

Shaver, K. G. y Scott, L. (1991). Person, process, and choice: The psychology of new venture creation. Entrepreneurship Theory and Practice, 16(2), 23-45.

Sheu, H. B., Lent, R. W., Brown, S. D., Miller, M. J., Hennessy, K. D. y Duffy, R. D. (2010). Testing the choice model of Social Cognitive Career Theory across Holland themes: A meta-analytic path analysis. Journal of Vocational Behavior, 76, 252-264.

Soutaris, V., Zerbinati, S. y Al-Laham, A. (2007). Do entrepreneurship programmes raise entrepreneurial intention of science and engineering students? The effect of learning, inspiration and resources. Journal of Business Venturing, 22, 566-591.

Stone, M. (1974). Cross-validatory choice and assessment of statistical predictions. Journal of the Royal Statistical Society, 36, 111-133.

Tang, M., Fouad, N. A. y Smith, P. L. (1999). Asian Americans' career choices: A path model to examine factors influencing their career choices. Journal of $V_{0}$ cational Behavior, 54, 142-199.

Tkachev, A. y Kolvereid, L. (1999). Self-employment intentions among Russian students. Entrepreneurship and Regional Development, 11, 269-280.

Toledano, N. y Urbano, D. (2008). Promoting entrepreneurial mindsets at universities: A case study in the South of Spain. European Journal of International Management, 2, 382-399.

Townsend, D. M., Busenitz, L. W. y Arthurs, J. D. (2010). To start or no to start: Outcome and ability expectations in the decision to start a new venture. Journal of Business Venturing, 25, 192-202.

Veciana, J. M., Aponte, M. y Urbano, D. (2005). University students' attitudes towards entrepreneurship: A two countries comparison. International Entrepreneurship and Management Journal, 1, 165-182.

Wilson, F., Kickul, J. y Marlino, D. (2007). Gender, entrepreneurial self-efficacy, and entrepreneurial career intentions: Implications for entrepreneurship education. Entrepreneurship Theory and Practice, 31, 387-406.

Wold, H. (1980). Soft modeling: Intermediate between traditional model building and data analysis. Mathematical Statistics, 6, 333-346.

Wold, H. (1982). Systems under indirect observation using PLS. En C. Fornell (Ed.), A Second Generation of Multivariate Analysis (pp. 325-347). Nueva York: Praeger Publishers.

Wold, H. (1985). Systems analysis by Partial Least Squares. En P. Nijkamp, H. Leitner y N. Wrigley (Ed.), Measuring the Unmeasurable (pp. 221-251). Dordrecht: Martinus Nijhoff Publishers.

Zhao, X., Lynch, J. G. y Chen, Q. (2010). Reconsidering Baron and Kenny: Myths and truths about mediation analysis. Journal of Consumer Research, 37, 197-206.

Zhao, H., Seibert, C. y Hills, C. (2005). The mediating role of self-efficacy in the development of entrepreneurial intentions. Journal of Applied Psychology, 90, 1265-1272.

Zellweger, T., Sieger, P. y Halter, F. (2011). Should I stay or should I go? Career choice intentions of students with family business background. Journal of Business Venturing, 26(5), 521-536.

(Artículo recibido: 25-10-2012; revisión recibida: 18-7-2013; aceptado: 2-9-2013) 